$3-7-2010$

\title{
Stability of Choice in The Honey Bee Nest-Site Selection Process
}

Andrew L. Nevai

University of Central Florida

Kevin M. Passino

Ohio State University

Parthasarathy Srinivasan

Cleveland State University, p.srinivasan35@csuohio.edu

Follow this and additional works at: https://engagedscholarship.csuohio.edu/scimath_facpub

Part of the Mathematics Commons

How does access to this work benefit you? Let us know!

\section{Repository Citation}

Nevai, Andrew L.; Passino, Kevin M.; and Srinivasan, Parthasarathy, "Stability of Choice in The Honey Bee Nest-Site Selection Process" (2010). Mathematics Faculty Publications. 232.

https://engagedscholarship.csuohio.edu/scimath_facpub/232

This Article is brought to you for free and open access by the Mathematics and Statistics Department at EngagedScholarship@CSU. It has been accepted for inclusion in Mathematics Faculty Publications by an authorized administrator of EngagedScholarship@CSU. For more information, please contact library.es@csuohio.edu. 


\title{
Stability of choice in the honey bee nest-site selection process
}

\author{
Andrew L. Nevai, Kevin M. Passino, Parthasarathy Srinivasan
}

\section{Introduction}

\subsection{Description of the honey bee nest-site selection process}

In honey bee (Apis mellifera) nest-site selection (reviewed in Seeley and Visscher, 2004; Seeley et al., 2006; Passino et al., 2008), a cluster is formed by the colony splitting itself when the queen and about half the old colony depart and assemble nearby, typically on a tree branch. To find a new home, "scout" bees from the swarm cluster begin to search a large area for a suitable new nest-site, typically the hollow of a tree. Scouts assess the quality of sites based on cavity volume, entrance height, entrance area, and other attributes that are likely correlated with colony success. Bees that find good sites return to the cluster and their initial "dance strength" (number of waggle runs, with each run communicating the angle and radial distance to the nest-site) is in proportion to the quality of the nest-site. Such bees will revisit the site many times, but each time they return to the cluster their dance strength decreases till they no longer dance. The number of recruits to each nest-site is in proportion to the number of dances for each site on the cluster. "Unemployed" scouts either rest or seek to observe dances. If they easily find a dancer they get recruited to a relatively high quality site. If they must wait too long to find a dancer, there are not many good nest-sites currently being assessed so they explore the environment for more sites. There is a quorum-sensing process at each nest-site, where once there is a certain number of bees at the site, the bees from that site "choose it" by returning to the cluster to prompt lift-off and then they guide the swarm to its new home (Beekman et al., 2006; Schultz et al., 2008). There is significant time-pressure to complete the nest-site selection process as fast as possible since weather and energy losses pose significant threats to an exposed colony. However, enough time must be dedicated to ensure that many bees can conduct independent evaluations of the site and establish a quorum at one that is likely to be the best site that the swarm has found. Hence, during nest-site selection the swarm strikes a balance between time minimization and site quality choice maximization.

\subsection{Modeling approaches}

The key experimental work in the area of honey bee nest-site selection is in Seeley and Buhrman (1999), Camazine et al. (1999), 
Seeley and Buhrman (2001), Seeley (2003), Seeley and Visscher (2003), and Seeley and Visscher (2004). There have also been a number of models published. First, the differential equation models introduced in Britton et al. (2002) examine whether bees need to make direct comparisons between the qualities of more than one site in order to make a decision (they need not). A discrete-time population matrix model introduced in Myerscough (2003) studies the ability of a swarm to produce a unanimous decision when dances reproduce and propagate according to a Leslie matrix. A simulation model that was validated for a range of experiments (those in Seeley and Buhrman, 1999; Camazine et al., 1999; Seeley and Buhrman, 2001; Seeley, 2003; Seeley and Visscher, 2003, 2004), and may be one of the most biologically realistic of all existing models, is used in Passino and Seeley (2006) to study the speed-accuracy trade-off in the choice process. In Perdriau and Myerscough (2007) the authors introduce a density-dependent Markov process model of honey bee nestsite selection and study the effects of site quality, competition between sites, and delays in site discovery. Next, the work in Janson et al. (2007) introduces an individual-based model and studies the swarm's scouting behavior and the impact of distance on choice. More recently, Marshall et al. (2009) compares models of nest-site selection to models of decision-making in vertebrate brains. Finally, we note that ant colonies performing nest-site selection have some broad similarities to the bees' nest-site selection (e.g., a speed-accuracy trade-off), and corresponding models and simulations have been developed (Mallon et al., 2001; Pratt et al., 2002; Franks et al., 2003; Pratt, 2005; Pratt et al., 2005).

The purpose of this paper is to develop a modeling approach for the honey bee nest-site selection process which lends itself to analytical methods. Our ordinary differential equation model still captures the essential dynamics of the nest-site selection process appearing in Britton et al. (2002) and the biologically realistic model in Passino and Seeley (2006), but in a way that still renders the model analytically tractable. Our model does not represent additional features of the nest-site selection process not considered, for instance, in Passino and Seeley (2006) (e.g., the impact of swarm size or distance of candidate nest-sites from the cluster). Yet, our model permits us to connect equilibrium analysis to the ability of a swarm to achieve quorum at a site in finite time. This provides significant insights into the dynamics of nest-site selection, the impact of site quality on quorum achievement, and influences on the outcome of competition between a lower quality site discovered first and a higher quality site discovered second. As these issues have not been examined in the modeling studies outlined above, we believe that our modeling approach can provide new clues into the mystery surrounding the honey bee nest-site selection process.

First, we introduce a pair of continuous-time compartment models in Section 2. The main theoretical results are stated in Section 3 and numerical results are presented in Sections 4 and 5 . In the concluding Discussion section we point out some ways in which the models can be made more inclusive (biologically realistic) and mention some open problems. Proofs for the theoretical results appear in the Appendix.

\section{Description of models}

Here we outline the basic structure of two models that we later use to study the honey bee nest-site selection process. The first model represents a swarm of bees deciding whether a site is viable (when no other sites are under consideration), and the second model characterizes a swarm selecting between two viable sites. Since bees that are not scouts play no discernable role in selecting a new home, we will make no distinction between bees and scouts unless stated otherwise.

\subsection{Assessment process for one site}

The assessment process (see Fig. 1 and Table 1) models nest-site selection dynamics after the one-time discovery of a single site by one bee and comprises movement between five compartments: resting (R), observation (O), exploration (E), assessment $(A)$, and dancing (D). We define bees in the first two compartments to be unemployed and all others to be employed. Let the continuous state variable $X(t)$ represent the fraction of bees in compartment $X \in\{R, O, E, A, D\}$ at time $t \geq 0$. We remark that if bees exit a compartment at rate $\alpha$ then $1 / \alpha$ represents the mean time spent in that compartment

Assumptions: We assume that resters become observers $(R \rightarrow O)$ at positive constant rate $a$, observers become employed $(O \rightarrow E, A)$ at positive constant rate $b$, explorers become observers $(E \rightarrow O)$ at positive constant rate $c$, site assessors become dancers $(A \rightarrow D)$ at positive constant rate $m$ and dancers cease to dance $(D \rightarrow R, A)$ at positive constant rate $n$. Let $p(D)=D /(D+e)$ be the fraction of newly employed bees which are successfully recruited to assess the site $(O \rightarrow A)$ when there are $D$ bees dancing for it, and let $q(D)=1-p(D)$ represent the remaining fraction $(O \rightarrow E)$. Here, the half-saturation constant $e$ is assumed to be positive. Also, a constant fraction $0<v \leq 1$ of bees that cease to dance immediately retire $(D \rightarrow R)$, while the remaining fraction $w=1-v$ return to the site to reassess it $(D \rightarrow A)$. All bees are initially resters, observers, and explorers except for a single assessor bee that has independently made a one-time discovery of

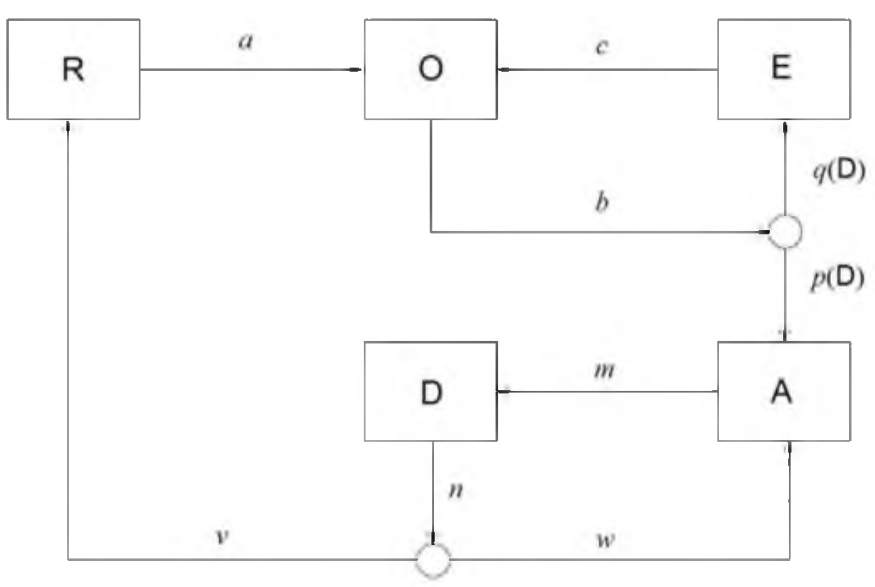

Fig. 1. Illustration of the assessment process for consideration of a single site.

Table 1

Quantities associated with the assessment process.

\begin{tabular}{ll}
\hline Notation & Meaning \\
\hline$R, O, E, A, D$ & $\begin{array}{l}\text { fraction of bees resting, observing, exploring, assessing, } \\
\text { and dancing } \\
\text { rates at which bees cease resting, observing, exploring, } \\
\text { assessing, and dancing } \\
\text { fraction of newly employed bees which become assessors } \\
\text { and explorers } \\
\text { fraction of bees that retire and return to site after dancing }\end{array}$ \\
$v(D), q(D)$ & \\
\hline
\end{tabular}


the site. These assumptions together imply that the governing system of equations is $(\dot{X} \stackrel{\text { def }}{=} d X / d t)$

$\dot{R}=-a R+v n D, \quad R(0)=R_{0}$,

$\dot{O}=a R-b O+c E, \quad O(0)=O_{0}$,

$\dot{E}=q(D) b O-c E, \quad E(0)=E_{0}$,

$\dot{A}=p(D) b O-m A+w n D, \quad A(0)=A_{0}$,

$\dot{D}=m A-n D, \quad D(0)=0$,

where $R_{0}+O_{0}+E_{0}+A_{0}=1$ and $0<A_{0} \ll 1$.

The influence of site quality: The description above does not identify what the role of site quality is on the assessment process. In nature, bees assess some sites to be of higher quality than others. Consequently, they will dance longer for better sites and return more often to them before retiring. We now indicate how some parameters in the assessment process can depend on site valuation (see Table 2 ). Suppose that every site can be assigned a value $Q \in(0,1)$ representing its overall quality relative to other sites, with $Q \rightarrow 0$ approaching an adverse site and $Q \rightarrow 1$ approaching a perfect site. We assume that site quality is uniformly sensed by every bee that assesses it (i.e., all bees judge the same site to have the same quality). Suppose that bees spend time $\sigma M Q$ in the dancing compartment before they retire or return to the site to reassess it. Here, the positive constant $\sigma$ represents the time required for a dancing bee to complete one waggle run and the positive constant $M$ is the maximum number of waggle runs performed by a bee dancing for a perfect site $(Q=1)$. It follows that the rate at which bees cease dancing is $n(Q)=(\sigma M Q)^{-1}$. To represent the fact that bees dancing for better sites are more likely to return to them before retiring, we assume that $v(Q)=1-Q$ and $w(Q)=Q$. Thus, dancing bees do not revisit adverse sites $(Q \rightarrow 0)$ and always revisit perfect sites $(Q \rightarrow 1)$. We will purposely exclude the limiting values of $Q=0$ and 1 from our analysis to ensure that $n(Q), v(Q)$, and $w(Q)$ are positive.

\subsection{Discrimination between two sites}

Let $\left(R, O, E^{n}, A, D^{\prime}\right)$ represent the state of the assessment process at time $t^{\circ}>0$, and suppose that at this time a second site is newly discovered. Then the assessment process gives way to a two-site discrimination process whose corresponding equations are (see Fig. 2)

$\dot{R}=-a R+\left\langle v_{k} n_{k} D_{k}\right\rangle, \quad R\left(t^{\prime}\right)=R$,

$\dot{O}=a R-b O+c E, \quad O\left(t^{\prime}\right)=O$,

$\dot{E}=q\left(D_{1}+D_{2}\right) b O-c E, \quad E\left(t^{\prime}\right)=E-A_{0}$,

$\dot{A}_{1}=\mu_{1}\left(D_{1}, D_{2}\right) p\left(D_{1}+D_{2}\right) b O-m A_{1}+w_{1} n_{1} D_{1}, \quad A_{1}(t)=A^{\prime}$,

$\dot{D}_{1}=m A_{1}-n_{1} D_{1}, \quad D_{1}(t)=D$,

Table 2

Parameters associated with the influence of site quality.

\begin{tabular}{ll}
\hline Notation & Meaning \\
\hline$Q$ & site quality \\
$\sigma$ & time spent on each waggle run \\
$M$ & number of waggle runs performed by bee \\
& dancing for perfect site \\
& rate at which bees cease dancing \\
& fraction of bees that retire and return to site \\
$v(Q), w(Q)$ & after dancing \\
\hline
\end{tabular}

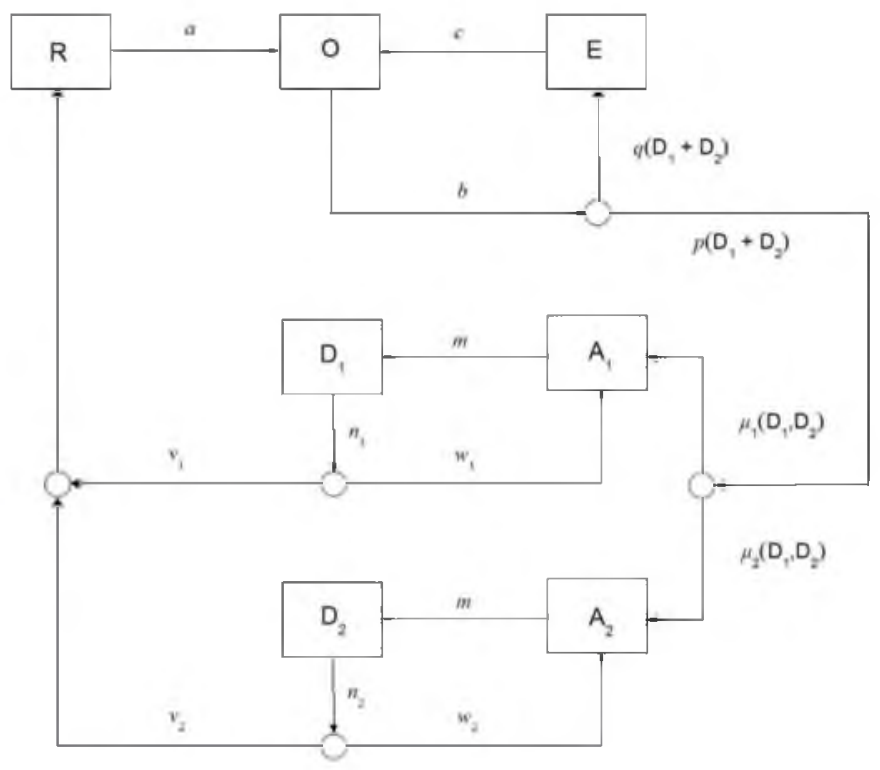

Fig. 2. Illustration of the discrimination process for consideration of two sites.

$A_{2}=\mu_{2}\left(D_{1}, D_{2}\right) p\left(D_{1}+D_{2}\right) b O-m A_{2}+w_{2} n_{2} D_{2}, \quad A_{2}\left(t^{\circ}\right)=A_{0}$

$D_{2}=m A_{2}-n_{2} D_{2}, \quad D_{2}\left(t^{\circ}\right)=0$

Here, $\mu_{k}\left(D_{1}, D_{2}\right)=D_{k} /\left(D_{1}+D_{2}\right)$ is the proportional fraction of newly recruited bees reporting to site $k=1,2$, the quantity $\left\langle v_{k} n_{k} D_{k}\right\rangle=v_{1} n_{1} D_{1}+v_{2} n_{2} D_{2}$ is the combined rate at which bees dancing for both sites retire, and $n_{k}=n\left(Q_{k}\right), v_{k}=v\left(Q_{k}\right)$, and $w_{k}=w\left(Q_{k}\right)$ depend on site quality $Q_{k}$ as described earlier. We remark that $\mu_{k}\left(D_{1}, D_{2}\right) p\left(D_{1}+D_{2}\right)=D_{k} /\left(D_{1}+D_{2}+e\right)$.

\section{Statement of main results}

Here we present our main analytical results. Proofs of the theorems are supplied in the Appendix. Short and informal proofs of their corollaries (what we call results) are given in the main text.

\subsection{Assessment model}

Our first main result concerns the solution of (2.1).

Theorem 1 (Existence of solution). A solution of (2.1) exists for all time. This solution is unique, nonnegative, and its components always sum to one.

We say that an equilibrium $\left(R^{*}, O^{*}, E^{*}, A^{*}, D^{*}\right)$ of $(2.1)$ is feasible if it is nonnegative and $R^{*}+O^{*}+E^{*}+A^{*}+D^{*}=1$. Define an equilibrium to be disinterested (DE) if $A^{*}=0$ and interested (IE) if $A^{*}>0$. Observe from the final equation of (2.1) that $D^{*}=0$ in a DE and $D^{*}>0$ in an IE. For a fixed constant quorum threshold $A_{q} \in(0,1)$, define an IE to be a partially interested equilibrium (PIE) if $A^{*}<A_{q}$ and a fully interested equilibrium (FIE) if $A^{*} \geq A_{q}$. It is clear from (2.1) that $(0, \bar{O}, \bar{E}, 0,0)$ is a $D E$, where we define

$\bar{O}=\frac{c}{b+c}$ and $\bar{E}=\frac{b}{b+c}$.

Next, observe from Fig. 1 that $p(D) b O$ represents the rate at which observer bees are recruited to the site and $\nu n D$ represents the rate at which dancing bees retire. In view of this remark, define the basic recruitment number to be the number of bees recruited by a 
single dancing bee in an otherwise disinterested equilibrium before it retires:

$\mathcal{R}_{0}=\left[\frac{\text { recruitment rate }}{\text { resting rate }}\right]_{\mathrm{DE}}=\lim _{D \rightarrow 0} \frac{p(D) b \bar{O}}{v n D}=\frac{p^{\prime}(0) b \bar{O}}{n v}=\frac{b \bar{O}}{e n v}$.

Then

$\mathcal{R}_{0}=\frac{1}{e n v}\left(\frac{1}{b}+\frac{1}{c}\right)^{-1}$

It is clear that $\mathcal{R}_{0}$ is a harmonic function of the rates at which observing bees become employed $(b)$ and explorer bees return to the swarm cluster $(c)$ and a monotone decreasing function of the recruitment constant $(e)$, the rate at which bees cease dancing $(n)$, and the fraction of them which retire $(v)$. The basic recruitment number $\mathcal{R}_{0}$ is analogous to the basic reproduction number of a disease model, in which resting bees, observing bees, and exploring bees are susceptible (healthy), assessing bees are infected but not infectious (latent), and dancing bees are both infected and infectious ( $\mathrm{N}$. Britton, personal communication). Define the absolute recruitment number to be the maximum possible recruitment rate for a single dancing bee (i.e., when all other bees are observers):

$\mathcal{B}_{0}=\left[\frac{\text { recruitment rate }}{\text { resting rate }}\right]_{0=1}=\lim _{D \rightarrow 0} \frac{p(D) b}{v n D}=\frac{p^{\prime}(0) b}{n v}=\frac{b}{e n v}$.

Since $\bar{O}<1$, it follows that $\mathcal{R}_{0}<\mathcal{B}_{0}$. Indeed, we would expect such an inequality to prevail because the recruitment rate is an increasing function of the fraction of bees that observe. The next result examines the feasibility and stability of the DE.

Theorem 2 (Disinterested equilibrium). A DE is always feasible and unique, and it has the form $(0, \bar{O}, \bar{E}, 0,0)$. If $\mathcal{R}_{0}<1$ then the $D E$ is locally asymptotically stable, but if $\mathcal{R}_{0}>1$ then it is unstable. Furthermore, if $\mathcal{B}_{0}<1$ then the DE is globally asymptotically stable.

Interestingly, the stability of the DE depends on neither the rate at which resting bees begin to observe $(a)$ nor the rate at which assessing bees begin to dance $(m)$. The next result concerns the situation in which the DE is unstable.

Theorem 3 (Interested equilibrium). An IE is feasible if and only if $\mathcal{R}_{0}>1$. When feasible, it is unique and it has the form

$R^{*}=\frac{n v}{a} D^{*}, \quad O^{*}=\frac{n v}{b}\left(D^{*}+e\right), \quad E^{*}=\frac{e n v}{c}, \quad A^{*}=\frac{n}{m} D^{*}$,

and

$D^{*}=\frac{\frac{1}{n v}-e\left(\frac{1}{b}+\frac{1}{c}\right)}{\left(\frac{1}{m}+\frac{1}{n}\right) \frac{1}{v}+\frac{1}{a}+\frac{1}{b}}$.

If, in addition, $a \geq c$ or $b+c \geq \max \{m, n\}$ then the IE is locally asymptotically stable.

First, it can be seen from (3.1), (3.2) with $\mathcal{R}_{0}>1$, and (3.3) that an IE always possesses fewer explorers $\left(E^{*}<\bar{E}\right)$ than a DE. Also, these equations and (3.4) together imply that an IE can possess either fewer observers $\left(O^{*}<\bar{O}\right)$ or more observers $\left(O^{*}>\overline{0}\right)$ than a $\mathrm{DE}$ depending on the relative values of $1 / n v$ and $1 / c-1 / a-1 / m v$. Second, it is not known in general whether the IE is stable when $a<c<\max \{m, n\}-b$. However, the inequality $a \geq c$ will always be satisfied in biologically realistic problems because it is reasonable to assume that on average scouts should spend far less time resting $(1 / a)$ than exploring $(1 / c)$. Finally, since

$A^{*}=\frac{\frac{1}{m v}-e\left(\frac{1}{b}+\frac{1}{c}\right) \frac{n}{m}}{\left(\frac{1}{m}+\frac{1}{n}\right) \frac{1}{v}+\frac{1}{a}+\frac{1}{b}}$ it is straightforward to determine whether an IE constitutes a PIE $\left(A^{*}<A_{q}\right)$ or an FIE $\left(A^{*} \geq A_{q}\right)$ for specific parameter values.

\subsection{Influence of site quality}

Recalling that $n(Q)=(\sigma M Q)^{-1}$ and $v(Q)=1-Q$, we observe from (3.2) that the basic recruitment number $\mathcal{R}_{0}$ strictly increases from 0 to $\infty$ as site quality $(Q)$ increases from 0 to 1 . It follows that there exists a critical site quality threshold $Q^{*}$ below which $\mathcal{R}_{0}<1$ and above which $\mathcal{R}_{0}>1$. This $Q^{*}$ satisfies $\mathcal{R}_{0}\left(Q^{*}\right)=1$, i.e.,

$\frac{\sigma M Q^{*}}{1-Q^{*}}=e\left(\frac{1}{b}+\frac{1}{c}\right) \Longrightarrow Q^{*}=\frac{e\left(\frac{1}{b}+\frac{1}{c}\right)}{e\left(\frac{1}{b}+\frac{1}{c}\right)+\sigma M}$

It is clear that $Q^{*} \in(0,1)$ and that $Q^{*}$ is a monotone increasing function of $e$ and a monotone decreasing function of $b, c, \sigma$, and $M$. As with the basic recruitment number, $Q^{*}$ depends on neither $a$ nor $m$. To summarize,

Result 1 (Influence of site quality on $\mathcal{R}_{0}$ ). There exists some $Q^{*} \in(0,1)$ such that $\mathcal{R}_{0}<1$ for $Q<Q^{*}$ and $\mathcal{R}_{0}>1$ for $Q>Q^{*}$.

The quantity $Q^{*}$ in Result 1 is a threshold below which a site cannot attract any interest at equilibrium $\left(A^{*}=0\right)$ and above which it can $\left(A^{*}>0\right)$. We now consider the $Q$-dependence of the equilibrium state variables. It can be seen by inspection or differentiation that $E^{*}$ in (3.3) and $D^{*}$ in (3.4) are monotone functions of $Q$, and that $R^{*}, O^{*}$, and $A^{*}$ in (3.3) have more complex $Q$-dependencies. To indicate that two quantities $A$ and $B$ share the same sign for all parameter values, we will write $A \longleftrightarrow B$. The influence of site quality on the IE is as follows.

Result 2 (Influence of site quality on IE). Let $Q>Q^{*}$, so that the IE is feasible. Then
(a) $\frac{d E^{*}}{d Q}<0$ and $\frac{d D^{*}}{d Q}>0$,
(b) $\frac{d R^{*}}{d Q} \div e\left(\frac{1}{b}+\frac{1}{c}\right)(1-Q)\left[\left(2 \sigma M+\frac{1}{m}-\frac{1}{a}-\frac{1}{b}\right) Q+\frac{1}{m}+\frac{1}{a}+\frac{1}{b}\right]$ $-\left(\sigma M+\frac{1}{m}\right) \sigma M Q^{2}$,
(c) $\frac{d O^{*}}{d Q}-e\left[\left(\frac{1}{b}+\frac{1}{c}\right)\left(\left(2 \sigma M+\frac{1}{m}-\frac{1}{a}-\frac{1}{b}\right) Q+\frac{1}{m}+\frac{1}{a}+\frac{1}{b}\right)(1-Q)\right.$ $\left.-\left(\left(\sigma M-\frac{1}{a}-\frac{1}{b}\right) Q+\frac{1}{m}+\frac{1}{a}+\frac{1}{b}\right)^{2}\right]-\left(\sigma M+\frac{1}{m}\right) \sigma M Q^{2}$,
(d) $\frac{d A^{*}}{d Q} \longrightarrow e\left(\frac{1}{b}+\frac{1}{c}\right)\left(\sigma M(2-Q) Q+\left(\frac{1}{a}+\frac{1}{b}\right)(1-Q)^{2}+\frac{1}{m}\right)$ $+\left(\frac{1}{a}+\frac{1}{b}-\sigma M\right) \sigma M Q^{2}$.

Two special cases of interest arise for the IE: one when the swarm is considering a site which is minimally interesting $\left(Q \rightarrow Q^{*}\right)$ and one when the site is close to perfect $(Q \rightarrow 1)$.

Result 3 (IE for minimally interesting site). In the limit as $Q \rightarrow Q^{*}$,

(a) $\left(R^{*}, O^{*}, E^{*}, A^{*}, D^{*}\right)=(0, \bar{O}, \bar{E}, 0,0)$,

(b) $\frac{d R^{*}}{d Q}>0, \quad \frac{d E^{*}}{d Q}<0, \quad \frac{d A^{*}}{d Q}>0$ and $\frac{d D^{*}}{d Q}>0$,

(c) $\frac{d O^{*}}{d Q}+\left(\frac{1}{c}-\frac{1}{a}-\frac{1}{m}\right) \sigma M-e\left(\frac{1}{b}+\frac{1}{c}\right)\left(\sigma M+\frac{1}{m}\right)$.

Thus, the IE approaches the DE as $Q \rightarrow Q^{*}$ and, depending on parameter values, the equilibrium fraction of observers can begin to either increase or decrease as site quality increases beyond $Q^{*}$. 
Result 4 (IE for perfect site). In the limit as $Q \rightarrow 1$,

(a) $\left(R^{*}, O^{*}, E^{*}, A^{*}, D^{*}\right)=\left(0,0,0, \frac{1}{1+m \sigma M}, \frac{m \sigma M}{1+m \sigma M}\right)$,

(b) $\frac{d R^{*}}{d Q}<0, \quad \frac{d O^{*}}{d Q}<0, \quad \frac{d E^{*}}{d Q}<0$ and $\frac{d D^{*}}{d Q}>0$,

(c) $\frac{d A^{*}}{d Q} \div e\left(\frac{1}{b}+\frac{1}{c}\right)\left(\sigma M+\frac{1}{m}\right)+\left(\frac{1}{a}+\frac{1}{b}-\sigma M\right) \sigma M$.

As the site approaches perfection, the IE approaches an equilibrium in which all bees are either assessing the site or dancing for it. Depending on parameter values, the equilibrium fraction of assessors can begin to increase or decrease as site quality decreases below one. It also follows from part (a) of Result 4 that an IE for a near perfect site $(Q \rightarrow 1)$ is an FIE if $A_{q} \leq(1+m \sigma M)^{-1}$ and it is a PIE otherwise. We now combine this observation with the properties of $A^{*}$ in Results 2-4.

Result 5 (Distinction between PIE and FIE). Let $Q>Q^{*}$, so that the IE is feasible, and suppose that $\sigma M<\frac{1}{a}+\frac{1}{b}$. If $A_{q} \leq(1+m \sigma M)^{-1}$ then there exists some $Q^{* *} \in\left(Q^{*}, 1\right]$ such that the IE is a PIE for $Q<Q^{* *}$ and an FIE for $Q \geq Q^{* *}$. However, if $A_{q}>(1+m \sigma M)^{-1}$ then the IE is always a PIE.

The quantity $\mathrm{Q}^{* *}$ in Result 5 is a threshold below which a site cannot attract a quorum at equilibrium $\left(A^{*}<A_{q}\right)$ and above which it can $\left(A^{*} \geq A_{q}\right)$. An explicit expression for $Q^{* *}$ can be obtained by solving the equation $A^{*}\left(Q^{* *}\right)=A_{q}$, where $A^{*}$ is as in (3.5). That is, we wish to solve

$\frac{1}{m v}-e\left(\frac{1}{b}+\frac{1}{c}\right) \frac{n}{m}=\left\{\left(\frac{1}{m}+\frac{1}{n}\right) \frac{1}{v}+\frac{1}{a}+\frac{1}{b}\right\} A_{q}$.

where $n=n\left(Q^{* *}\right)$ and $v=v\left(Q^{* *}\right)$. We multiply both sides by $v$ and then substitute $n=\left(\sigma M Q^{* *}\right)^{-1}$ and $v=1-Q^{* *}$ to obtain

$\frac{1}{m}-e\left(\frac{1}{b}+\frac{1}{c}\right) \frac{1-Q^{* *}}{m \sigma M Q^{* *}}=\left\{\frac{1}{m}+\frac{1}{n}+\left(\frac{1}{a}+\frac{1}{b}\right)\left(1-Q^{* *}\right)\right\} A_{q}$.

Rearrangement produces a quadratic equation $\omega_{2}\left(Q^{* *}\right)^{2}+$ $\omega_{1} Q^{* *}+\omega_{0}=0$ with coefficients

$\omega_{2}=A_{q}\left(\frac{1}{a}+\frac{1}{b}\right) \sigma M$,

$\omega_{1}=\frac{1}{m}\left\{\sigma M+e\left(\frac{1}{b}+\frac{1}{c}\right)\right\}-A_{q}\left\{\frac{1}{a}+\frac{1}{b}+\frac{1}{m}+\frac{1}{n}\right\} \sigma M$,

$\omega_{0}=-\frac{e}{m}\left(\frac{1}{b}+\frac{1}{c}\right)$

Since the discriminant $\Delta=\omega_{1}^{2}-4 \omega_{2} \omega_{0}$ is always positive, there are two real roots,

$Q_{ \pm}^{* *}=\frac{-\omega_{1} \pm \sqrt{\omega_{1}^{2}-4 \omega_{2} \omega_{0}}}{2 \omega_{2}}$.

Furthermore, since $\Delta>\omega_{1}^{2}$, it follows that there is exactly one positive root which we call $Q^{* *}$. Whether $Q^{* *} \leq 1$ depends on parameter values in a complicated manner.

\subsection{Discrimination model}

In the two-site discrimination model, there are four kinds of equilibrium states: a disinterested equilibrium (DE) in which all bees are either observing $\left(O^{*}=\bar{O}\right)$ or exploring $\left(E^{*}=\bar{E}\right)$, two semiinterested equilibria in which bees show interest for only one site, and co-interested equilibrium states in which bees show interest for both sites.
We treat here the stability of the DE only. As in the assessment process, each site $(k=1,2$ ) has a basic recruitment number when it is in isolation $\left(\mathcal{R}_{0}^{k}\right)$. It is also possible to define a system-wide basic recruitment number $\left(\mathcal{R}_{0}^{*}\right)$ as a measure of the DE's sensitivity to dancing for either site. Following van den Driessche and Watmough (2002), we write the discrimination process as $\dot{x}=(\mathcal{F}-\mathcal{V})(x)$, where

$$
\begin{aligned}
& x=\left(\begin{array}{c}
R \\
O \\
A_{1} \\
D_{1} \\
A_{2} \\
D_{2}
\end{array}\right), \quad \mathcal{F}(x)=\left(\begin{array}{c}
0 \\
0 \\
\mu_{1}\left(D_{1}, D_{2}\right) p\left(D_{1}+D_{2}\right) b O \\
0 \\
\mu_{2}\left(D_{1}, D_{2}\right) p\left(D_{1}+D_{2}\right) b O \\
0
\end{array}\right) \text { and } \\
& \mathcal{V}(x)=\left(\begin{array}{c}
a R-v_{1} n_{1} D_{1}-v_{2} n_{2} D_{2} \\
-a R+b O-c E \\
m A_{1}-w_{1} n_{1} D_{1} \\
-m A_{1}+n_{1} D_{1} \\
m A_{2}-w_{2} n_{2} D_{2} \\
-m A_{2}+n_{2} D_{2}
\end{array}\right) \text {. }
\end{aligned}
$$

We do not include an equation for $E$ on account of the relation $R+O+E+A_{1}+D_{1}+A_{2}+D_{2}=1$. The linearizations of $\mathcal{F}$ and $\mathcal{V}$ evaluated at the $D E$, respectively, are block-triangular matrices

$(D \mathcal{F})_{*}=\left(\begin{array}{ll}0 & 0 \\ 0 & F\end{array}\right)$ and $(D \mathcal{V})_{*}=\left(\begin{array}{cc}A & B \\ 0 & V\end{array}\right)$

where

$A=\left(\begin{array}{cc}a & 0 \\ -a+c & b+c\end{array}\right), \quad B=\left(\begin{array}{cccc}0 & -v_{1} n_{1} & 0 & -v_{2} n_{2} \\ c & c & c & c\end{array}\right)$. $F=\left(\begin{array}{cc|cc}0 & b \overline{\mathrm{O}} / e & & \\ 0 & 0 & & \\ \hline & & 0 & b \overline{\mathrm{O}} / e \\ & & 0 & 0\end{array}\right)$,

$V=\left(\begin{array}{cc|cc}m & -w_{1} n_{1} & & \\ -m & n_{1} & & \\ \hline & & m & -w_{2} n_{2}\end{array}\right)$.

Since the eigenvalues of $A$ are positive, it follows that the DE will be locally stable if and only if the eigenvalues of the blockdiagonal matrix $F-V$ have negative real part. According to van den Driessche and Watmough (2002), this condition is equivalent to the eigenvalues of $F V^{-1}$ lying within the unit circle. A straightforward calculation reveals that $\rho\left(F V^{-1}\right)=\max \left\{\mathcal{R}_{0}^{1}, \mathcal{R}_{0}^{2}\right\}$. The following result summarizes these remarks.

Result 6 (Disinterested equilibrium). Let $\mathcal{R}_{0}^{*}=\max \left\{\mathcal{R}_{0}^{1}, \mathcal{R}_{0}^{2}\right\}$. If $\mathcal{R}_{0}^{*}<1$ then the DE is locally asymptotically stable, but if $\mathcal{R}_{0}^{*}>1$ then it is unstable.

\section{Numerical study of the assessment model}

In this section we explore numerically the assessment process and consider the role of site quality on quorum time.

\subsection{Preliminaries}

We say that a site is viable at equilibrium if $Q \geq Q^{* *}$ because then $A^{*} \geq A_{q}$. But in nature, bees will only select a site capable of attracting a quorum in finite time (Seeley and Visscher, 2004). In view of this observation, define a site to be viable in finite time (or simply viable) if it is able to attract a quorum in finite time, 
i.e., $A(t)=A_{q}$ for some $t>0$. When a site is viable, define its quorum time to be $t_{q}=\min \left\{t \geq 0: A(t)=A_{q}\right\}$. If a site is not viable, then its quorum time is undefined. Since bees in nature must also balance the accuracy of their search with speed (Passino and Seeley, 2006), we define a site to be viable in $\tau$ hours if $t_{q}$ is defined and $t_{q} \leq \tau$. Finally, define the site viability threshold to be $Q=\inf \{Q \in[0,1]:$ a site of quality $Q$ is viable $\}$ and the related quantity

$Q(\tau)=\inf \{Q \in[0,1]:$ a site of quality $Q$ is viable in $\tau$ hours $\}$.

Since it is not known in general whether $A(t)$ is always monotone, we recognize that a site may in principle be viable (in $\tau$ hours) even if it is not viable at equilibrium $\left(A^{*}<A_{q}\right)$. Furthermore, a site viable at equilibrium $\left(A^{*} \geq A_{q}\right)$ may not be viable in $\tau$ hours. The latter situation could occur, for example, if $t_{q}$ exists but $t_{q}>\tau$.

In the numerics below, we allow site quality $Q$ to vary against a background of otherwise fixed parameter values (see Table 3). We also assume that the assessment process begins "one bee from the DE", that is, $(R, O, E, A, D)(0)=\left(0, \bar{O}, \bar{E}-A_{0}, A_{0}, 0\right)$ with $0<A_{0} \ll 1$. We now argue that it is natural to implement this starting condition. A swarm that has not yet discovered a site will consist entirely of resters, observers, and explorers, say at time $s<0$. It is not hard to see from Fig. 1 that the DE is globally asymptotically stable with respect to all such initial conditions, i.e., those satisfying $A(s)=D(s)=0$. Consequently, moments before the first site is discovered, the swarm will be close to the disinterested equilibrium, and discovery of that site amounts to moving one explorer bee $\left(A_{0}\right)$ into the assessment compartment with the distribution of the other bees being unchanged.

\subsection{Behavior of the model}

Illustrated in Fig. 3 are four simulations of the assessment process for different choices of site quality $Q$. It can be seen that when $Q \leq Q^{*}$ (upper left panel) then an IE does not exist and the system quickly settles down to the DE. When $Q \in\left(Q^{*}, Q^{* *}\right)$ (upper right panel) then the system approaches a PIE $\left(0<A^{*}<A_{q}\right)$. When $Q=Q^{* *}$ (lower left panel) than the system is attracted to a minimal FIE $\left(A^{*}=A_{q}\right)$ and if $Q>Q^{* *}$ then the system approaches an FIE with $A^{*}>A_{q}$. Fig. 4 illustrates the same simulations but portrays more clearly the behavior of $A(t)$. In particular, we see that quorum time is essentially infinite when $Q=Q^{* *}$ (lower left panel) and finite when $Q>Q^{* *}$ (lower right panel).

\subsection{Quorum time versus site quality}

Fig. 5 illustrates the monotone relationship between quorum time and site quality. Observe that the bifurcation curve is a decreasing function of $Q$ whose slope is increasing and that the critical site quality threshold $Q^{* *}$ at which a site can attract a quorum at equilibrium provides an excellent approximation to $Q(50)$, the $Q$-value at which the bifurcation curve intersects the horizontal line $t_{q}=50$. We conjecture that in general $Q^{* *}$ is equal to the site viability threshold $Q$ (i.e., the vertical asymptote of the graph).

\section{Numerical study of the two-site discrimination model}

We now turn to the two-site discrimination process.

\subsection{Preliminaries}

When a swarm considers two sites simultaneously its attention is necessarily divided between them and thus the time to quorum for either site is slowed. Obviously, if the two sites are found at the same time, then the higher quality site will always be chosen provided that there is sufficient time for it to attract a quorum. However, if the higher quality site (say $Q_{2}$ ) is found after the site with lower quality $\left(Q_{1}\right)$, then the difference in discovery times $\left(t^{\circ}\right)$ plays a crucial role in determining which site is chosen. Thus, we are interested in the outcome of "competition" between two sites that differ in their discovery times $\left(t^{\prime}\right)$, their quality $\left(\Delta Q=Q_{2}-Q_{1}\right)$, or both.

The possible outcomes of the discrimination process are as follows: (i) no-decision or failure (quorum achieved at neither site), (ii) site 1 chosen (quorum achieved at site 1 but not site 2 or at site 1 before site 2), (iii) site 2 chosen (quorum achieved at site 2 but not site 1 or at site 2 before site 1), and (iv) split-decision (quorum achieved at both sites simultaneously).

In the simulations which follow, parameter values are always as in Table 3 and the initial condition is always the result of "one bee from the DE" for the associated assessment process. That is, $\left(R, O, E, A_{1}, D_{1}, A_{2}, D_{2}\right)\left(t^{*}\right)=\left(R, O, E^{\circ}-A_{0}, A, D, A_{0}, 0\right)$ where $\left(R^{*}, O^{\prime}\right.$,

Table 3

Parameter values used in all simulations. Composite parameters (those defined in terms of other parameters) are also listed.

\begin{tabular}{|c|c|c|c|}
\hline Parameter & Value & Units & Rationale \\
\hline a & 0.100 & $\min ^{-1}$ & $10 \mathrm{~min}$ resting \\
\hline$b$ & 0.125 & $\min ^{-1}$ & $8 \mathrm{~min}$ observing \\
\hline$c$ & 0.033 & $\min ^{-1}$ & 30 min exploring \\
\hline$m$ & 0.050 & $\min ^{-1}$ & $20 \mathrm{~min}$ assessing \\
\hline$\sigma$ & 0.1 & $\min$ & consecutive waggle runs start $6 s$ apart \\
\hline$M$ & 150 & - & 150 waggle runs for a perfect site Seeley and Buhrman (1999) \\
\hline$e$ & 0.323 & - & chosen so that $Q^{*}=0.450$ \\
\hline$Q$ & $(0,1)$ & - & varied between simulations \\
\hline$n$ & $0.066 / Q$ & $\min ^{-1}$ & composite parameter \\
\hline$v$ & $1-Q$ & - & composite parameter \\
\hline$w$ & $Q$ & - & composite parameter \\
\hline$A_{0}$ & 0.005 & - & 200 scout bees \\
\hline$A_{q}$ & 0.1 & - & 20 assessing bees produce quorum Seeley and Visscher $(2003,2004)$ \\
\hline$\overline{0}$ & 0.211 & - & composite parameter \\
\hline $\bar{E}$ & 0.789 & - & composite parameter \\
\hline$Q^{*}$ & 0.450 & - & composite parameter $\left(A^{*}=0\right)$ \\
\hline$Q^{* *}$ & 0.500 & - & composite parameter $\left(A^{*}=A_{q}\right)$ \\
\hline$t^{*}$ & {$[0,3000]$} & $\min$ & varied between simulations \\
\hline
\end{tabular}



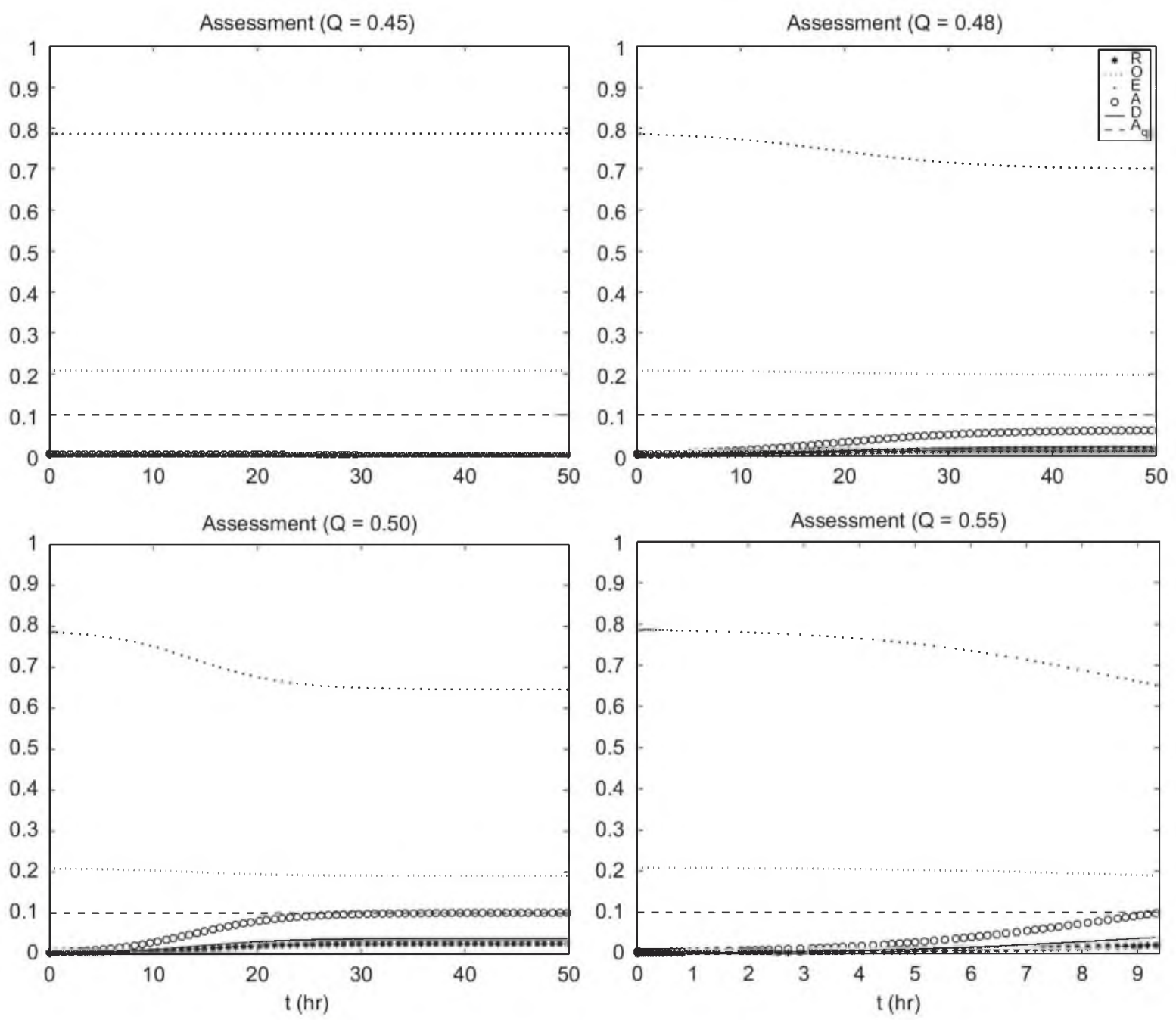

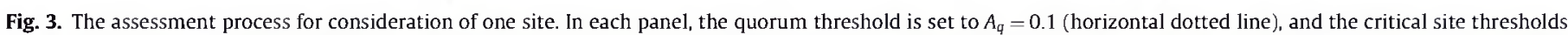

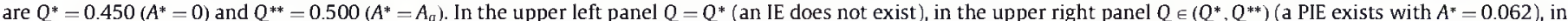

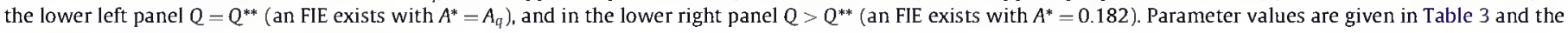
initial conditions are "one bee from the DE".

$\left.E^{n}, A, D^{\prime}\right)$ is the state of the assessment process at time $t^{\circ}$ after starting "one bee from the DE".

\subsection{Behavior of the model}

Figs. 6 and 7 illustrate the discrimination process in which a lower quality site $(Q=0.6)$ is discovered before a higher quality site $(Q=0.7)$. According to Fig. 5, the lower quality site in isolation can attract a quorum in about $6.2 \mathrm{~h}$. However, it can be seen from both figures that the lower quality site's ability to attract a quorum can be considerably slowed by the presence of the higher quality site and that the difference in discovery times strongly influences the identity of the chosen site. In Fig. 6 the second site is discovered $2 \mathrm{~h}$ after the first site and the higher quality site attracts a quorum first $(6.658 \mathrm{~h})$. In Fig. 7 the second site is discovered $35 \mathrm{~min}$ later, enabling the lower quality site to attract a quorum first $(7.168 \mathrm{~h})$.

\subsection{Fixed difference in discovery times}

The outcome of the discrimination process is illustrated in Fig. 8 for two sites discovered simultaneously $\left(t^{\circ}=0\right)$ and at different times $\left(t^{\circ}=1,2,3\right)$. In each plot, neither site is viable in the lower left quadrant $\left(Q_{1}, Q_{2}<Q\right)$, only site 1 is viable in the lower right quadrant $\left(Q_{1}>Q\right.$ and $\left.Q_{2}<Q\right)$, only site 2 is viable in the upper left quadrant $\left(Q_{1}<Q\right.$ and $\left.Q_{2}>Q\right)$, and both sites are viable in the upper right quadrant $\left(Q_{1}, Q_{2}>Q\right)$.

We consider first the case in which both sites are discovered simultaneously ( $t^{\circ}=0$ ). If neither site is viable, then neither will be selected. If only one site is viable, then it will always be the one selected. Finally, when both sites are viable, then the higher quality site will always be selected and a split-decision line is clearly visible.

Next, suppose that the difference in site discovery times is $t^{\circ}=1 \mathrm{~h}$. This time, when both sites are viable, the split-decision curve bends sharply upward because site 1 has an advantage in time over site 2 . For larger differences in discovery times $\left(t^{\circ}=2\right.$ and $3 \mathrm{~h}$ ), the split-decision curve bends upward more steeply.

In the event that a site must be chosen within $\tau$ hours (after which the discrimination process terminates regardless of the outcome), then distraction between sites can produce a band of no-decision around the split-decision curve. The width of this nodecision band increases as the urgency of the discrimination process increases, where urgency is negatively related to the quorum cut-off time $\tau$. For a $50 \mathrm{~h}$ time limit, the resulting band is too narrow to be seen. 

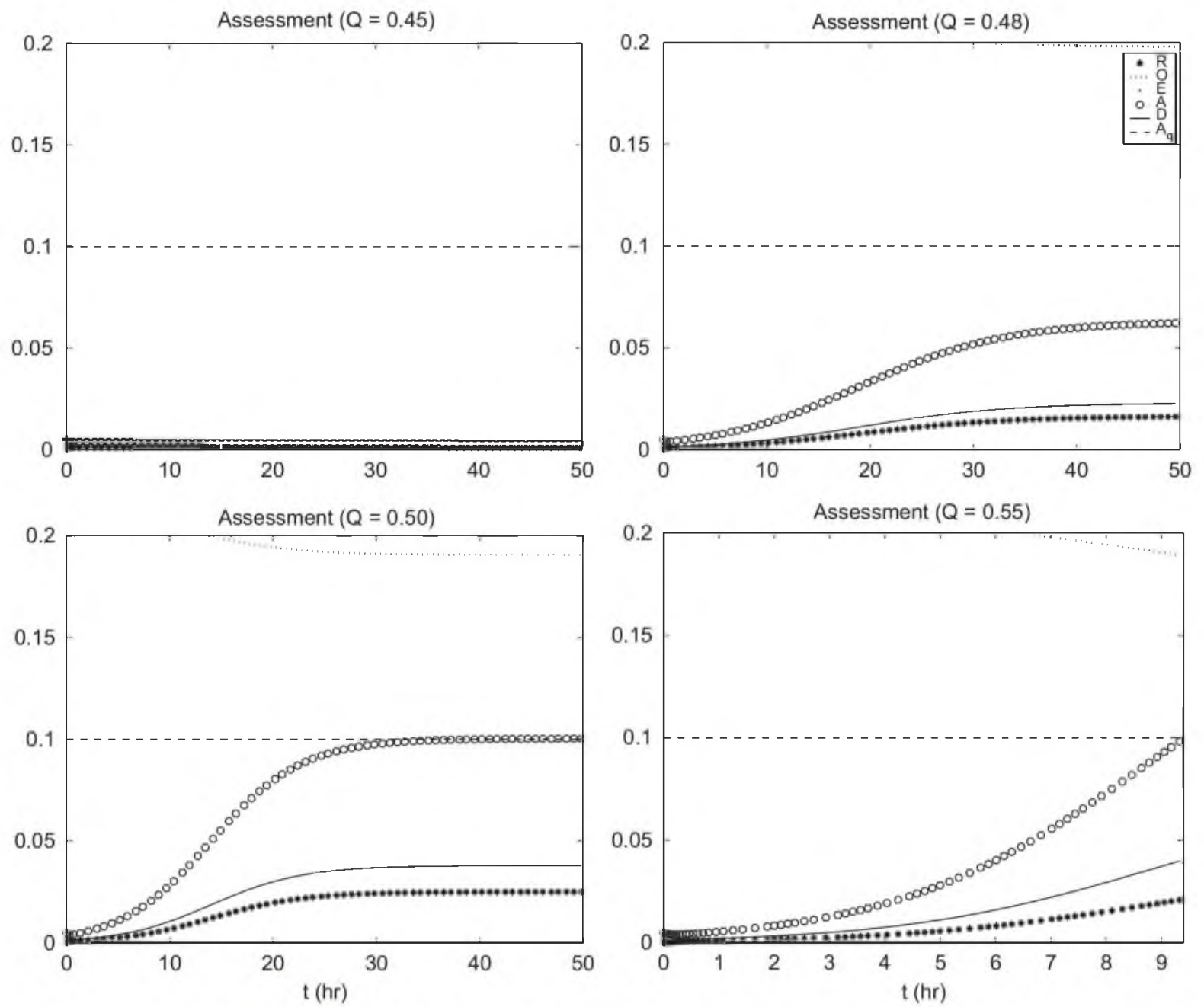

Fig. 4. The same panels as in Fig. 3 but with the vertical axis scaled differently. Quorum is achieved in the lower right panel at $t_{q}=9.450 \mathrm{~h}$.

\subsection{Fixed difference in site quality}

Next, we consider the influence of site quality difference on the outcome of the discrimination process.

Consider two sites with a fixed difference in site quality, say $\Delta Q=0.2$ as in Fig. 9 (lower left panel). In the leftmost region (to the left of the leftmost vertical line) neither site is viable and so neither site is chosen. In the middle region (between the vertical lines) only site 2 is viable and it will always be the one to attract a quorum. However, if the discrimination process is urgent, then site 2 is viable within the resulting time limit only if it is discovered sufficiently fast. Here, site 2 can attract a quorum in $50 \mathrm{~h}$ below the dotted curve but not above it. The dotted curve rises as it extends to the rightmost vertical line, which is to be expected because higher quality sites can attract quorums faster (and are more likely to do so in $50 \mathrm{~h}$ ) than lower quality sites. In the rightmost region (to the right of the rightmost vertical line) both sites are viable. Here, site 2 attracts a quorum first below the split-decision curve and site 1 attracts a quorum first above it. Larger differences in site quality $(\Delta Q)$ increase (1) the width of the middle region, (2) the asymptotic value of $t^{\circ}$ in the middle region and (3) the area in the rightmost region within which site 2 can be chosen.

Again, a band of no-decision exists around the split-decision curve when the process is urgent. However, this band is too narrow to be seen for a $50 \mathrm{~h}$ time limit.

\section{Discussion}

We now make some concluding remarks concerning the onesite assessment process and two-site discrimination process, including the statement of some open problems. We also mention some of the many ways in which the two models can be made more realistic from a biological standpoint.

\subsection{One-site assessment process}

Our numerical simulations of the assessment model suggest that when the DE is locally stable $\left(\mathcal{R}_{0}<1\right)$, then it is always globally attracting. Since our Theorem 2 establishes the latter result only when $\mathcal{B}_{0}<1$, it remains to extend the result to a global one when $\mathcal{R}_{0}<1$.

Although the force of recruitment $p(D)=D /(D+e)$ was selected to have a saturating form, no hard data exists to support this specific form, or indeed any form. To analyze the sensitivity of the model to the choice of the recruitment function, we suggest that its results be compared to one implementing frequency-dependence, say $p(D)=D /(D+O+R)$. We suspect that for the latter function, the stability results obtained for the DE may become global, but at a significant cost - in that the analysis of the IE will be much more difficult. 


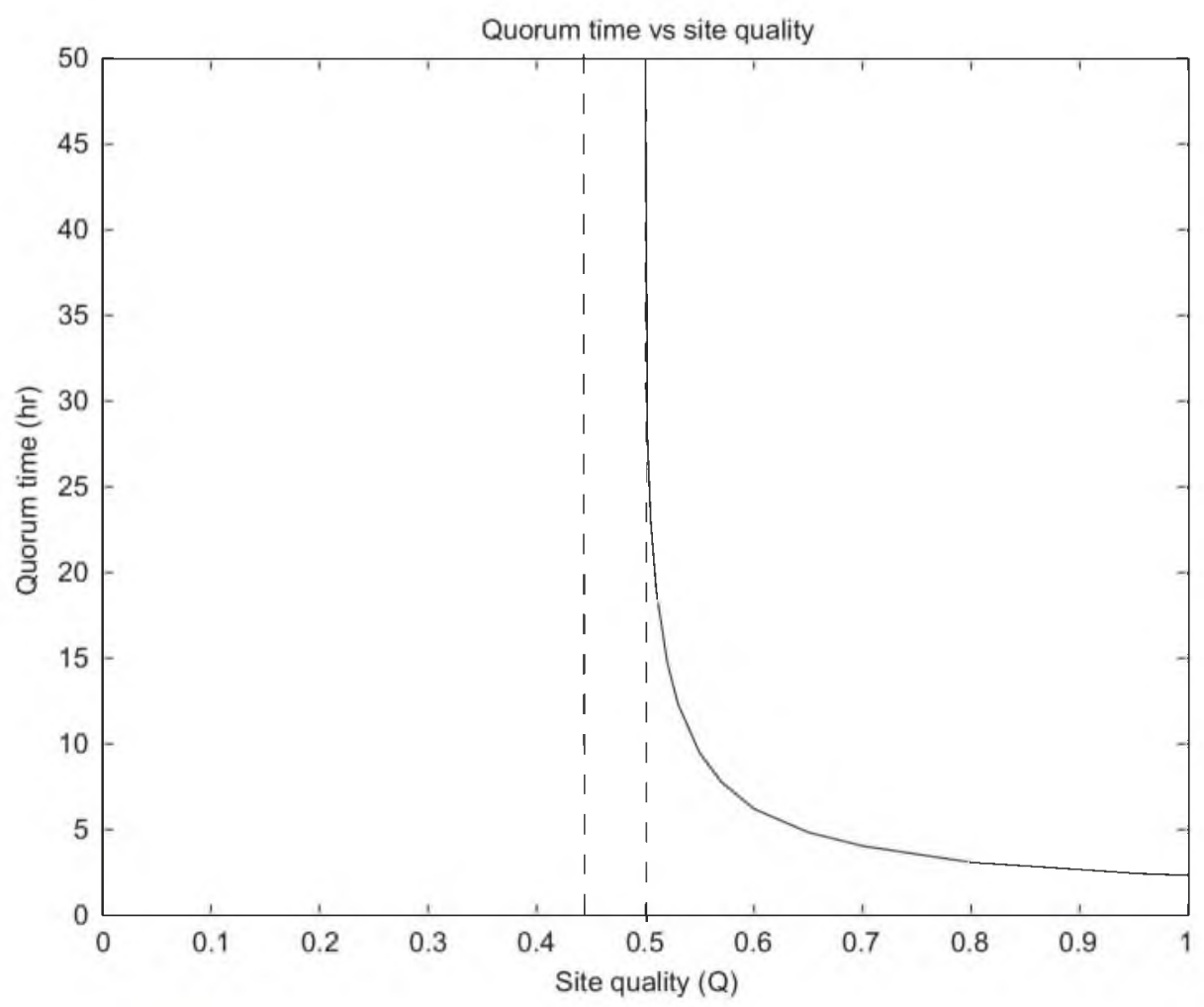

Fig. 5. Quorum time $\left(t_{\sigma}\right)$ versus site quality $(Q)$. The two vertical lines are at $Q^{*}=0.450$ (left) and $Q^{* *}=0.500$ (right). Parameter values are given in Table 3 and the initial condition is always "one bee from the DE".
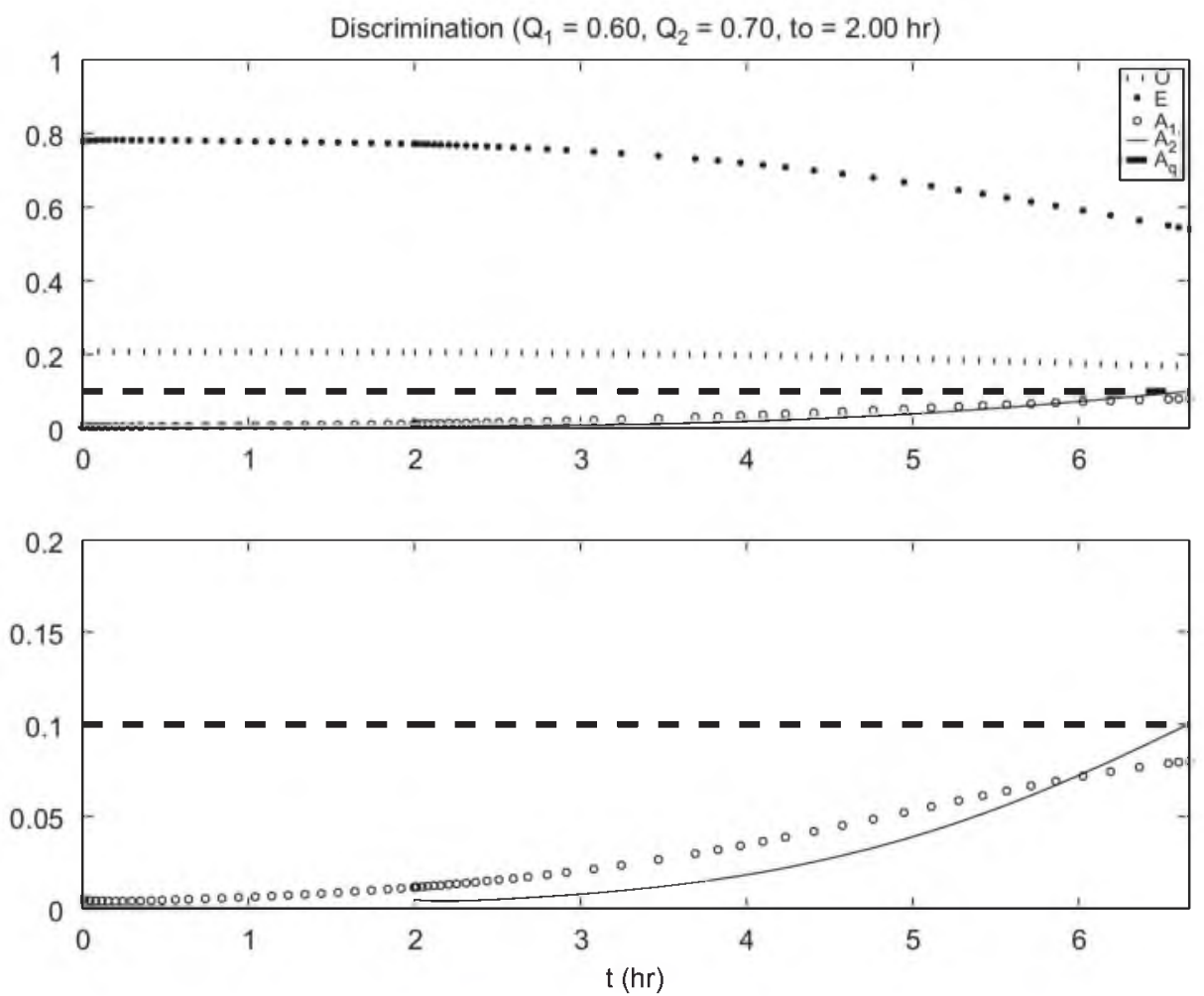

Fig. 6. The discrimination process for consideration of two sites. The quorum threshold is set to $A_{q}=0.1$ (horizontal dotted line) and the difference in discovery times is $t^{\circ}=2 \mathrm{~h}(120 \mathrm{~min})$. In the upper panel only $O, E_{1} A_{1}$, and $A_{2}$ are shown. The same simulation is shown in the lower panel but the vertical axis is scaled differently and only $A_{1}$ and $A_{2}$ are shown. Quorum is reached by site 2 in $6.683 \mathrm{~h}$. Parameter values are given in Table 3 and the initial condition is the result of "one bee from the DE" for the associated assessment process. 

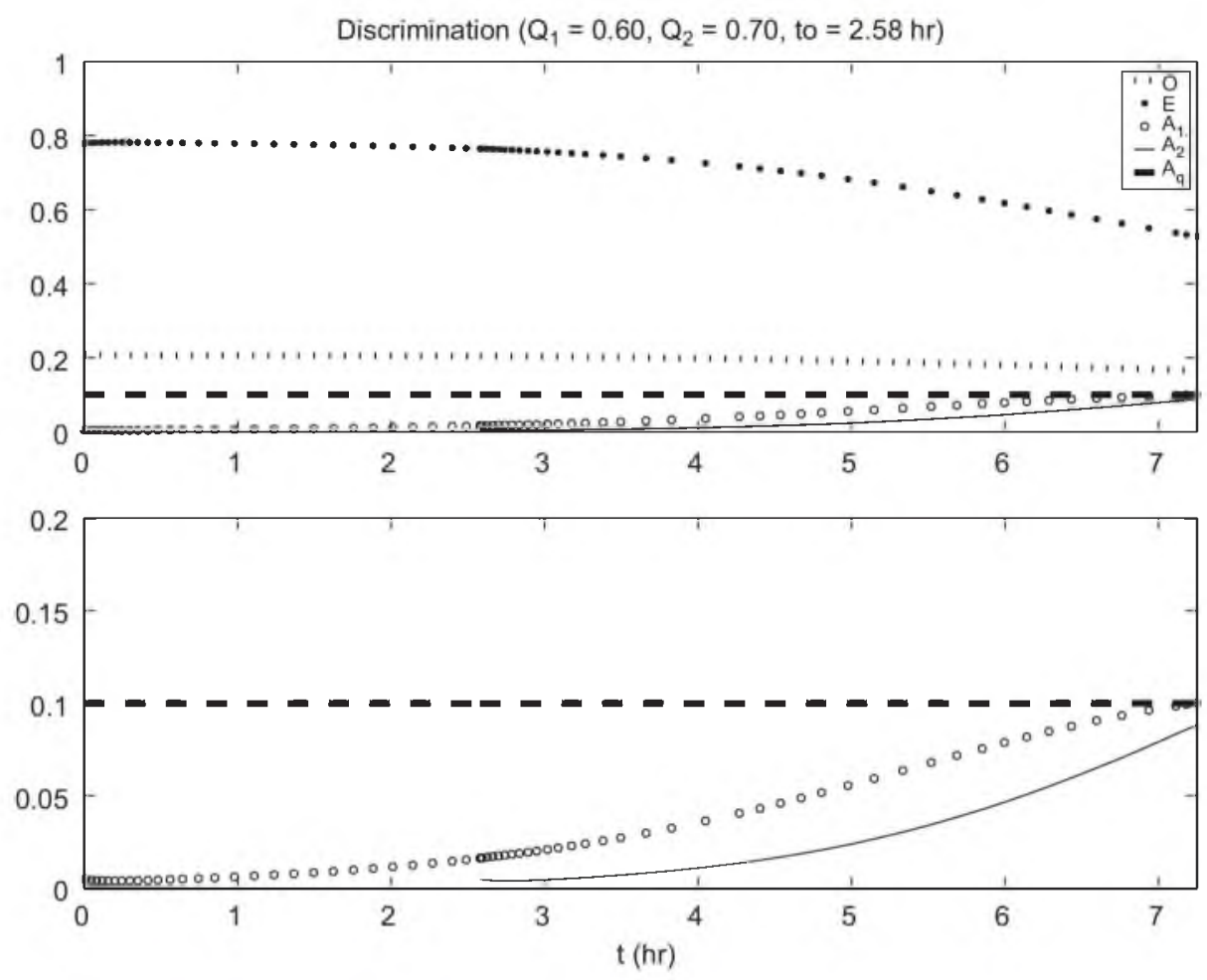

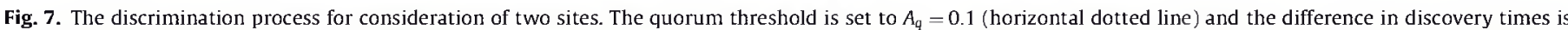

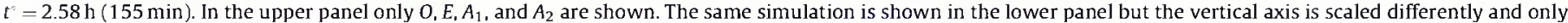

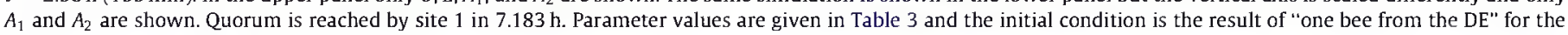
associated assessment process.
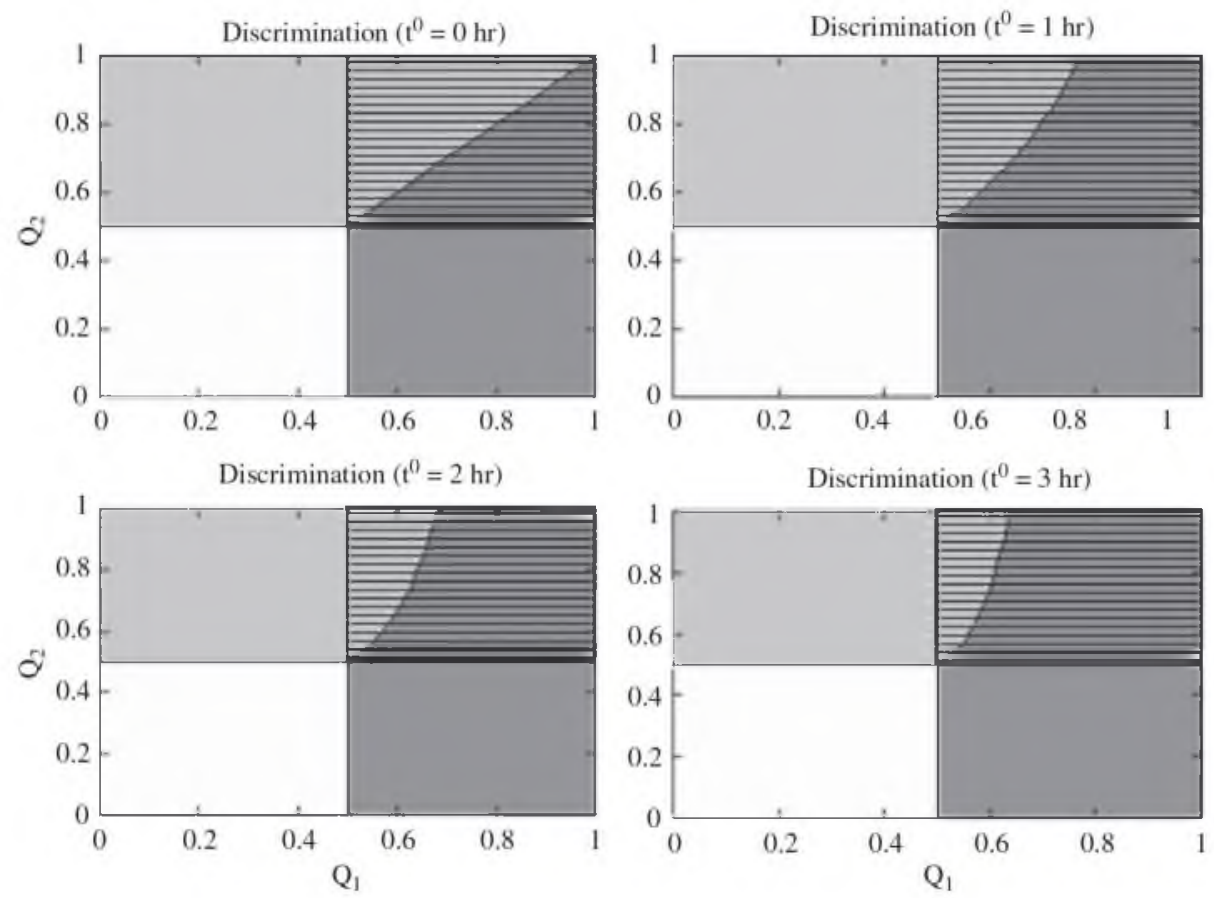

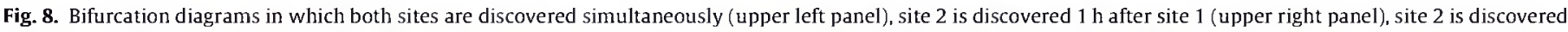

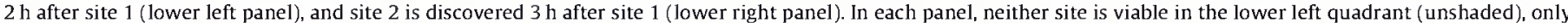

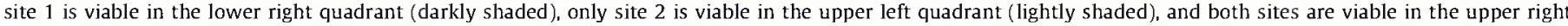

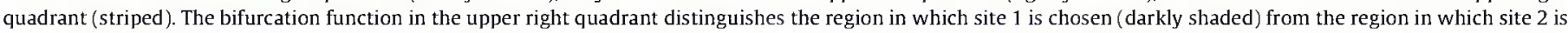

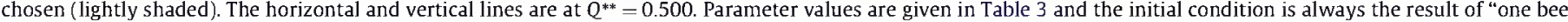
from the DE" for the associated assessment process. 

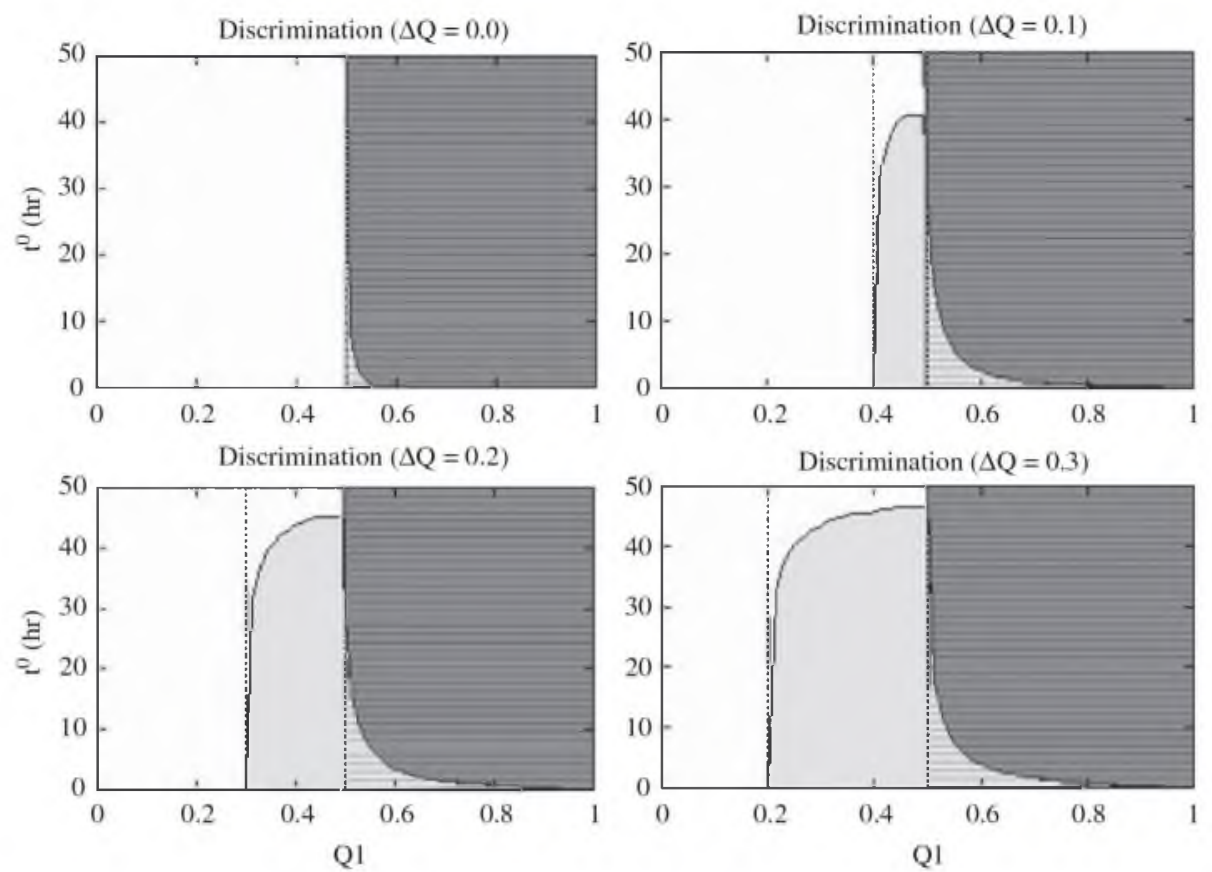

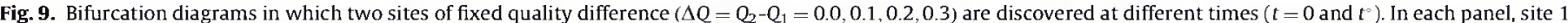

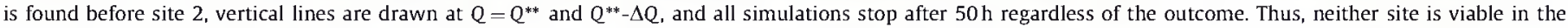

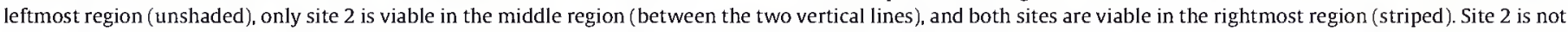

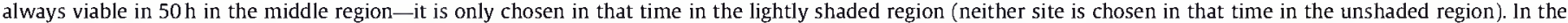

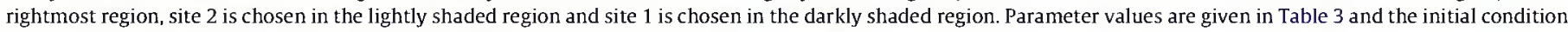
is always the result of "one bee from the DE" for the associated assessment process.

It is likely that the IE is stable whenever it is feasible $\left(\mathcal{R}_{0}>1\right)$. However, we only established the local stability of the IE for cases in which $a \geq c$ or $b+c \geq \max \{m, n\}$. The former condition almost certainly holds for biologically realistic situations because bees spend less time resting $(1 / a)$ than exploring $(1 / c) .{ }^{1}$ However, the second inequality presents no ready reason for it to hold. Indeed, we allow it to hold only so that $\xi_{0}$ is nonnegative in the proof of Theorem 3. Although an IE can never be globally asymptotically stable, it can in principle attract all initial conditions different from the DE. Our numerical simulations suggest that this is indeed the case whenever the IE is feasible, yet a proof of this result has remained elusive.

\subsection{Two-site discrimination process}

The discrimination model characterizes a swarm whose attention is divided between two competing sites. Although the linear stability of the disinterested equilibrium is known from Result 6, it is not known what parameter conditions would lead to the semi- and co-equilibrium states being stable. Furthermore, when they are stable, it would be desirable to know how large their regions of attractions are.

As mentioned earlier, a band of indecision exists around the split-decision curve, and this band increases in width as the urgency of the decision process increases. It remains to quantify this relationship in some rigorous manner, i.e., to determine the width of the band as a function of the quorum cut-off time $\tau$. Although establishing such a relationship analytically may be difficult, perhaps it can be done so numerically.

\footnotetext{
${ }^{1}$ Experiments have not been conducted to show that this is the case: however, this conclusion is made based on personal observations of KMP for three swarms conducting nest-site selection.
}

As in other nest-site selection models (Britton et al., 2002; Passino and Seeley, 2006), bees recruited to one site cannot be recruited to another until they have completed their assessment and dancing for the first site. Consequently, when bees dance for a small number of observers, then very few bees will ultimately be recruited. However, unlike other models (e.g., Passino and Seeley, 2006), our discrimination process is not subject to the effects of a finite pool size (in which no observer may be present at times) because no state variable can ever become zero in finite time. Nevertheless, the state variable representing observers can become so small that it represents less than one (discrete) bee, in which case it has effectively become zero.

\subsection{Biological realism}

In order to obtain a mathematically tractable model, it was necessary to ignore a number of features of the nest-site selection process and to produce modeling approximations for others. First, in nature the process is driven by discrete bees that make individual decisions in an asynchronous fashion (e.g., bees arrive and depart at the cluster and nest-sites at random times) (Seeley and Buhrman, 1999; Camazine et al., 1999; Seeley and Buhrman, 2001; Seeley, 2003; Seeley and Visscher, 2003, 2004). Here, however, there is no randomness in the occurrence of arrival/ departure events over time, and the number of bees is represented by a continuous variable (as in, e.g., Britton et al., 2002). Individual decisions are represented by the functional relationships developed for the differential equations (unlike the model in Passino and Seeley, 2006). Our consideration of a pair of related models (assessment and discrimination) is a consequence of the fact that bees in nature discover sites asynchronously, and so newly discovered sites must compete for the bees' attention with sites already under consideration. This requires careful attention be paid to the initial condition of the assessment model, and allowing its state at some future time to form the initial 
condition of the discrimination model (save for the location of one explorer bee). The nest-site selection process typically occurs on the time scale of about one to three days (Seeley and Buhrman, 1999; Camazine et al., 1999; Seeley and Buhrman, 2001; Seeley, 2003; Seeley and Visscher, 2003, 2004), yet we ignore changes associated with deaths of scouts that occurs at random times. The model in Passino and Seeley (2006) included the effects of deaths, which is mainly a slow "leakage" of bees out of the process that has no effect on discrimination as it seems that deaths occur independent of the quality of a nest-site.

Next, dancing induces a distributed nonlinear feedback whereby observer bees become recruited to different sites (Passino and Seeley, 2006). Since waggle runs are performed in pairs (or figureeights) followed by a frantic run to another part of the cluster (Seeley and Buhrman, 1999; Camazine et al., 1999; Seeley and Buhrman, 2001; Seeley, 2003; Seeley and Visscher, 2003, 2004), the parameter $\sigma$ must account for both of these times. The number of dances in a "bout," which represents the number of times that a bee will dance for a potential nest-site before resting, is really an integer determined by site quality. Also, the number of waggle runs performed in each dance decreases in a linear manner independent of site quality (Seeley, 2003). Here, we model recruitment via a process that has the number of dances decrease exponentially. A full model of the dance-recruitment process (e.g., as in Passino and Seeley, 2006) results in an explosion in the number of states (states are needed to represent the first, second, etc. times of visits to each candidate nest-site) and a resulting loss of analytical tractability.

In nature, the distance of a site from the swarm cluster may affect both its discovery time and quorum time. For instance, the decision process may favor a site of reasonably good quality which is very close, to a better site which is much farther away (there are, however, no existing experimental studies on the effect of the distance to candidate nest-sites on the dynamics of the nest-site selection process). Currently, the discrimination process accounts for site distance only through the difference in discovery times, and does not account for any additional time required by recruits in visiting sites that are farther away. No other models cited in the Introduction consider explicitly the effects of distance either.

Finally, since the assessment model is deterministic, every bee must necessarily sense the same value of $Q$ for a fixed site (as in Britton et al., 2002). The model can be made more realistic by allowing bees to sample site quality from a distribution with fixed mean and variance (as in Passino and Seeley, 2006). In such a stochastic model, it would be interesting to see how the mean and variance of site quality influence a swarm's decision to select a site in finite time - something not yet studied in the modeling and analysis literature.

In a future work, we hope to use our model to address related and important (yet unsolved) problems in the area of honey bee nest-site selection, including: how well do colonies deal with asynchronous discovery, how might they quantitatively tune their behavior to adjust the speed/accuracy tradeoff, and how might they adjust their behavior to avoid deadlock when no nest-site is particularly good.

\section{Acknowledgments}

The authors would like to thank Thomas D. Seeley for many informative discussions on the honey bee nest-site selection process, Nick Britton for suggestions on calculating the basic recruitment number, and two anonymous referees for their helpful comments. This material is based upon work supported by the National Science Foundation under Agreement No. 0112050 .

\section{Appendix A. Proofs of theorems}

\section{A.1. Proof of theorem 1 - existence of solution}

First, we observe from (2.1) that $d(R+O+E+A+D) / d t=0$, and since $(R+O+E+A+D)(0)=1$, we conclude that $(R+O+E+$ $A+D)(t)=1$ for as long as the solution exists.

Next, we show that there exists some $T>0$ such that $X(t)>0$ for $X \in\{R, O, E, A, D\}$ and $0<t<T$. To begin, recall that $X(0) \geq 0$ for $X \in\{R, O, E\}, \quad A(0)>0, \quad D(0)=0, \quad$ and $\quad D^{\prime}(0)=m A(0)-n D(0)=$ $m A(0)>0$. Thus, there exists $\varepsilon_{1}>0$ such that $A(t)>0$ and $D(t)>0$ for $0<t<\varepsilon_{1}$. If $R(0)>0$, then it is clear that there exists $\varepsilon_{2}<\varepsilon_{1}$ such that $R(t)>0$ for $0<t<\varepsilon_{2}$. On the other hand, if $R(0)=0$, then $R^{\prime}(0)=-a R(0)+v n D(0)=0$ and $R^{\prime \prime}(0)=-a R^{\prime}(0)+$ $v n D^{\prime}(0)>0$. Again, there exists $\varepsilon_{2}<\varepsilon_{1}$ such that $R(t)>0$ for $0<t<\varepsilon_{2}$. Next, if $O(0)>0$, then obviously there exists $\varepsilon_{3}<\varepsilon_{2}$ such that $O(t)>0$ for $0<t<\varepsilon_{3}$. However, if $O(0)=0$, then $O^{\prime}(0)=a R(0)-b O(0)+c E(0)=a R(0)+c E(0) \geq 0$. Since $R(0)+E(0)+$ $A(0)=1$ and $A(0) \ll 1$, it must be that either $R(0)>0$ or $E(0)>0$. In either case, $O^{\prime}(0)>0$ and there exists $\varepsilon_{3}<\varepsilon_{2}$ such that $O(t)>0$ for $0<t<\varepsilon_{3}$. Finally, if $E(0)>0$, then we may find $\varepsilon_{4}<\varepsilon_{3}$ such that $E(t)>0$ for $0<t<\varepsilon_{4}$. But, if $E(0)=0$, then $E^{\prime}(0)=$ $q(D(0)) b O(0)-c E(0)=b O(0) \geq 0$. If $E^{\prime}(0)>0$, then there exists $\varepsilon_{4}<\varepsilon_{3}$ such that $E(t)>0$ for $0<t<\varepsilon_{4}$. Suppose now that $E^{\prime}(0)=0$. Then $O(0)=0$ and as argued above $O^{\prime}(0)>0$. Moreover, $E^{\prime \prime}(0)=q^{\prime}(D(0)) D^{\prime}(0) b O(0)+q(D(0)) b O^{\prime}(0)-c E^{\prime}(0)=b O^{\prime}(0)>0$. Again, there exists $\varepsilon_{4}<\varepsilon_{3}$ such that $E(t)>0$ for $0<t<\varepsilon_{4}$. We conclude that $X(t)>0$ for $X \in\{R, O, E, A, D\}$ and $0<t<T$, where $T$ is taken to be $\varepsilon_{4}$.

Now, we show that $0 \leq X(t) \leq 1$ for $X \in\{R, O, E, A, D\}$ and $t \geq 0$, with equality possible only when $t=0$. We may assume that $\varepsilon_{1}$, $\varepsilon_{2}, \varepsilon_{3}$, and $\varepsilon_{4}$ are chosen to be maximal above, and therefore that $T$ is also maximal. Since $X(t)>0$ for $X \in\{R, O, E, A, D\}$ and $0<t<T$ and $(R+O+E+A+D)(t)=1$ for $0<t<T$, it must be that $X(t)<1$ for $X \in\{R, O, E, A, D\}$ and $0<t<T$. Let $X \in\{R, O, E, A, D\}$ and $\alpha=\max \{a . b, c, m . n\}$. Since $X^{\prime}(t)>-\alpha X$ for $0<t<T$, it must be that $0<X(T) \leq 1$. Since this inequality holds for every $X$, it cannot be that $X(T)=1$. That is, $0<X(T)<1$. Since $X$ is bounded, the maximality of $T$ implies that $T=\infty$. Thus, $0<X(t)<1$ for $t>0$. Since $0 \leq X(0) \leq 1$, we conclude that $0 \leq X(t) \leq 1$ for $t \geq 0$, with equality possible only when $t=0$.

It remains only to establish uniqueness of the solution. Since the five state variables always sum to one, we may eliminate the equation for one variable (say $E$ ) to obtain the reduced inhomogeneous system:

$$
\frac{d}{d t}\left(\begin{array}{l}
R \\
O \\
A \\
D
\end{array}\right)=\left(\begin{array}{cccc}
-a & 0 & 0 & n v \\
a-c & -b-c & -c & -c \\
0 & 0 & -m & n w \\
0 & 0 & m & -n
\end{array}\right)\left(\begin{array}{l}
R \\
O \\
A \\
D
\end{array}\right)+\left(\begin{array}{c}
0 \\
c \\
p(D) b O \\
0
\end{array}\right) .
$$

Let $B$ be the square matrix in the equation above, $x=(R, O, A, D)^{t}$, and $h(x)=(0, c, p(D) b O, 0)^{t}$. It suffices to show that $F(x)=B x+h(x)$ is bounded and Lipschitz on $\Omega=\left\{x \in \mathbb{R}^{4}: x \geq 0\right.$ and $\left.\|x\|_{1} \leq 1\right\}$, where $\|x\|_{1}=R+O+A+D$. The boundedness of $F$ on $\Omega$ is immediate. To distinguish between the components of $x$ and $y$, let us write $x=\left(R_{x}, O_{x}, A_{x}, D_{x}\right)^{t}$ and $y=\left(R_{y}, O_{y}, A_{y}, D_{y}\right)^{t}$. In the Euclidean norm, $\quad\|F(x)-F(y)\| \leq\|B(x-y)\|+\|h(x)-h(y)\| \leq$ $\|B\|\|x-y\|+b\left|p\left(D_{x}\right) O_{x}-p\left(D_{y}\right) O_{y}\right|$. It follows that $\|F(x)-F(y)\| \leq$ $\|B\|\|x-y\|+b p\left(D_{x}\right)\left|O_{x}-O_{y}\right|+b\left|p\left(D_{x}\right)-p\left(D_{y}\right)\right| O_{y}$. Since $p(D)=D /(D+$ $e$ ) and $O_{y}$ are upper bounded by unity on $\Omega$ and the former has Lipschitz constant $1 / e$, we conclude that $\|F(x)-F(y)\| \leq\|B\|\|x-y\|+$ $b\left|O_{x}-O_{y}\right|+(b / e)\left|D_{x}-D_{y}\right|$. Thus, $\|F(x)-F(y)\| \leq[\|B\|+b+(b / e)]\|x-y\|$ and $F$ is Lipschitz on $\Omega$. This completes the proof. 


\section{A.2. Proof of theorem 2 the disinterested equilibrium}

Since $p(0)=0$ and $q(0)=1$, it is straightforward to verify that $(0, \bar{O}, \bar{E}, 0,0)$ is always a feasible $D E$ and that it is unique. To determine its local stability, it is helpful to write system (2.1) as the reduced system (A.1) in the proof of Theorem 1. Linearizing around the $\mathrm{DE}$ results in the Jacobian matrix

$$
\begin{aligned}
& J^{*}=\left(\begin{array}{cc|cc}
-a & 0 & 0 & n v \\
a-c & -b-c & -c & -c \\
\hline & & -m & n w \\
& & m & -n
\end{array}\right) \\
& +\left(\begin{array}{l|lc} 
& & \\
\hline & 0 & b \overline{\mathrm{O}} / e \\
0 & 0
\end{array}\right) .
\end{aligned}
$$

Here, we used the fact that $p(0)=0$ and $p^{\prime}(0)=1 /$ e. A simple calculation shows that the upper block-triangular matrix $J^{*}$ has eigenvalues

$\lambda\left(J^{*}\right)=-a,-b-c, \frac{-m-n \pm \sqrt{m^{2}-2 m n+n^{2}+4 m(n w+b \bar{O} / e)}}{2}$.

These eigenvalues all have negative real part if and only if $n w+b \bar{O} / e<n$. Recalling that $v+w=1$, we see that the $\mathrm{DE}$ is locally asymptotically stable if $b \bar{O}<e n v$ (i.e., $\mathcal{R}_{0}<1$ ) and it is unstable if the inequality is reversed.

To prove global stability of the $\mathrm{DE}$, suppose that $\mathcal{B}_{0}<1$ and recall that $X(t) \in(0,1)$ for $X \in\{R, O, E, A, D\}$ and $t>0$. Define a partial ordering on $\mathbb{R}^{2}$ by $(\sigma, \tau) \leq(\hat{\sigma}, \hat{\tau})$ if $\sigma \leq \hat{\sigma}$ and $\tau \leq \hat{\tau}$.

First, we show that $R(t), A(t), D(t) \rightarrow 0$ as $t \rightarrow \infty$. Define the functions $x(t), y(t)$, and $z(t)$ by the linear system

$\dot{x}=f(x, y, z) \stackrel{\text { def }}{=}-a x+v n z$,

$\dot{y}=g(x, y, z) \stackrel{\text { def }}{=}(b / e) z-m y+w n z$,

$\dot{z}=h(x, y, z) \stackrel{\text { def }}{=} m y-n z$.

Assume that $x(0)=R(0), y(0)=A(0)$, and $z(0)=D(0)$. The origin is locally asymptotically stable in (A.2) if the Jacobian matrix

$J^{*}=\left(\begin{array}{ccc}-a & 0 & v n \\ 0 & -m & (b / e)+w n \\ 0 & m & -n\end{array}\right)$

is stable. The characteristic equation of $J^{*}$ is

$(\lambda+a)\left[\left(\lambda^{2}+(m+n) \lambda+m(n-(b / e)-w n)\right]=0\right.$,

and since a quadratic polynomial $\lambda^{2}+r \lambda+s$ is stable if and only if $r$ and $s$ are both positive, it follows that $J^{*}$ is stable if and only if

$n-w n>b / e \Longleftrightarrow v n>b / e \Longleftrightarrow b<e n v \Longleftrightarrow \mathcal{B}_{0}<1$.

Here, we used the fact that $v+w=1$. Since we assumed that $\mathcal{B}_{0}<1$, it must be that the origin is locally asymptotically stable in (A.2). As (A.2) is a linear system, the origin is in fact globally asymptotically stable in (A.2). Finally, it is easily checked from (2.1) and (A.2) that

1. $\dot{R}=f(R, A, D), \dot{A} \leq g(R, A, D)$, and $\dot{D}=h(R, A, D)$.

2. if $(\xi, \theta) \leq(\vec{\xi}, \hat{\theta})$ then $f(\eta, \xi, \theta) \leq f(\eta, \hat{\xi}, \hat{\theta})$.

3. if $(\eta, \theta) \leq(\hat{\eta}, \hat{\theta})$ then $g(\eta, \xi, \theta) \leq g(\hat{\eta}, \hat{\xi}, \hat{\theta})$.

4. if $(\eta, \xi) \leq(\hat{\eta}, \hat{\xi})$ then $h(\eta, \xi, \theta) \leq h(\hat{\eta}, \hat{\xi}, \theta)$.

We conclude from Theorem B1 in Smith and Waltman (1995) that $R(t) \leq x(t), \quad A(t) \leq y(t)$, and $D(t) \leq z(t)$ for $t \geq 0$. Since $x(t), y(t), z(t) \rightarrow 0$ as $t \rightarrow \infty$, it must be that $R(t), A(t), D(t) \rightarrow 0$ as $t \rightarrow \infty$.
We now show that $O(t) \rightarrow \bar{O}$ and $E(t) \rightarrow \bar{E}$ as $t \rightarrow \infty$. For $\varepsilon \geq 0$, consider the forced linear system

$\dot{x}_{\varepsilon}=f_{\varepsilon}\left(x_{\varepsilon}, y_{\varepsilon}\right) \stackrel{\text { def }}{=} a \varepsilon-b x_{\varepsilon}+c y_{\varepsilon}$,

$\hat{y}_{\varepsilon}=g_{\varepsilon}\left(x_{\varepsilon}, y_{\varepsilon}\right) \stackrel{\text { def }}{=} q(\varepsilon) b x_{\varepsilon}-c y_{\varepsilon}$.

Recall that $q(\varepsilon)=1-p(\varepsilon)=e /(\varepsilon+e)$. If $\varepsilon=0$ then let $\delta>0$ and set $x_{0}(\delta)=O(\delta)>0$ and $y_{0}(\delta)=E(\delta)>0$. For $\varepsilon>0$ there exists $T_{\delta}>0$ such that $R(t), D(t) \in(0, \varepsilon)$ for $t \geq T_{\varepsilon}$. In this case, set $x_{\varepsilon}\left(T_{\varepsilon}\right)=$ $O\left(T_{\varepsilon}\right)>0$ and $y_{\varepsilon}\left(T_{\varepsilon}\right)=E\left(T_{\varepsilon}\right)>0$. Let us write (A.3) as

$\dot{z}_{\varepsilon}=A_{\varepsilon} z_{\varepsilon}+u_{\varepsilon}$

where we define

$z_{\varepsilon}=\left(\begin{array}{l}x_{i} \\ y_{\varepsilon}\end{array}\right), \quad A_{\varepsilon}=\left(\begin{array}{cc}-b & c \\ q(\varepsilon) b & -c\end{array}\right)$ and $u_{\delta}=\left(\begin{array}{c}a \varepsilon \\ 0\end{array}\right)$.

Suppose first that $\varepsilon>0$. Then

$z_{\varepsilon}^{*}=-A_{i}^{-1} u_{i}=\left(\begin{array}{c}a(e+\varepsilon) / b \\ a e / c\end{array}\right)$

is an equilibrium of (A.4) and it is unique. Since $A_{\varepsilon}$ is stable, we have that $z_{i}^{*}$ is globally asymptotically stable in (A.4) (to see this, let $\theta_{\varepsilon}=z_{\varepsilon}-z_{\varepsilon}^{*}$ and consider the resulting equation $\left.\theta_{\varepsilon}=A_{\varepsilon} \theta_{\varepsilon}\right)$. Thus, $x_{\varepsilon}(t) \rightarrow a(e+\varepsilon) / b$ and $y_{\varepsilon}(t) \rightarrow a e / c$ as $t \rightarrow \infty$. Moreover, there exists $\tilde{T}_{\varepsilon} \geq T_{\varepsilon}$ such that $y_{\varepsilon}(t)>0$ for $t \geq \tilde{T}_{\varepsilon}$. For the case $\varepsilon=0$, we have $\dot{x}_{0}=-b x_{0}+c y_{0}$ and $\dot{y}_{0}=b x_{0}-c y_{0}$. Since $\dot{x}_{0}+\dot{y}_{0}=0$, we let $\rho=x_{0}(\delta)+y_{0}(\delta)$ to obtain $\dot{x}_{0}=-b x_{0}+c\left(\rho-x_{0}\right)=-(b+c) x_{0}+c \rho$. This equation possesses a unique and explicit solution

$x_{0}(t)=\frac{c \rho+\left((b+c) x_{0}(\delta)-c \rho\right) \exp (-(b+c)(t-\delta))}{b+c}$.

Also, $y_{0}(t)=\rho-x_{0}(t)$ and therefore $x_{0}(t) \rightarrow c \rho /(b+c)$ and $y_{0}(t) \rightarrow b \rho /(b+c)$ as $t \rightarrow \infty$. Next, it is easily checked from (2.1) and (A.3) that

1. $f_{0}(O, E) \leq \dot{O} \leq f_{n}(O, E)$ for $t \geq T_{t}$.

2. $g_{0}(O, E) \geq \dot{E} \geq g_{i}(O, E)$ for $t \geq T_{i}$.

3. if $\bar{\xi} \leq \hat{\xi}$ then $f_{i}(\eta, \hat{\xi}) \leq f_{i}(\eta, \hat{\xi})$.

4. if $\eta \leq \hat{\eta}$ then $g_{s}(\eta, \xi) \leq g_{\varepsilon}(\hat{\eta}, \xi)$.

Again, we conclude from Theorem B1 in Smith and Waltman (1995) that $x_{0}(t) \leq O(t) \leq x_{\varepsilon}(t)$ and $y_{0}(t) \geq E(t) \geq y_{\varepsilon}(t)$ for $t \geq \max \left\{\delta, T_{\varepsilon}\right\}$. It follows then that $x_{0}(t) / y_{0}(t) \leq O(t) / E(t) \leq$ $x_{\varepsilon}(t) / y_{\varepsilon}(t)$ for $t \geq \max \left\{\delta, \tilde{T}_{\varepsilon}\right\}$. Since $x_{0}(t) / y_{0}(t) \rightarrow c / b$ and $x_{\varepsilon}(t) /$ $y_{\varepsilon}(t) \rightarrow c(e+\varepsilon) / b e$ as $t \rightarrow \infty$, it must be that $c / b \leq \liminf _{t \rightarrow \infty} O(t)$ $E(t) \leq \limsup _{t \rightarrow \infty} O(t) / E(t) \leq c(e+\varepsilon) /$ be. Letting $\varepsilon \rightarrow 0$, it follows that $O(t) / E(t) \rightarrow c / b$ as $t \rightarrow \infty$. Since $(R+O+E+A+D)(t) \equiv 1$ and $R(t), A(t), D(t) \rightarrow 0$, we find that $O(t)+E(t) \rightarrow 1$ as $t \rightarrow \infty$. Thus, $O(t) \rightarrow c /(b+c)=\bar{O}$ and $E(t) \rightarrow b /(b+c)=\bar{E}$ as $t \rightarrow \infty$ and the theorem is proved.

\section{A.3. Proof of theorem 3 - the interested equilibrium}

We first show that an IE exists if and only if $\mathcal{R}_{0}>1$. The equilibrium equations are obtained from (2.1) by setting the time derivatives equal to zero,

$0=-a R^{*}+v n D^{*}$

$0=a R^{*}-b O^{*}+c E^{*}$

$0=q\left(D^{*}\right) b O^{*}-c E^{*}$,

$0=p\left(D^{*}\right) b O^{*}-m A^{*}+w n D^{*}$,

$0=m A^{*}-n D^{*}$. 
It is immediately clear from the first and last equations that $R^{*}=(n v / a) D^{*}$ and $A^{*}=(n / m) D^{*}$, and from the third equation that $c E^{*}=q\left(D^{*}\right) b O^{*}$. Substituting these results into the second equation and rearranging produces

$b O^{*}=n v \times \frac{D^{*}}{p\left(D^{*}\right)}$,

Since $p\left(D^{*}\right)=D^{*} /\left(D^{*}+e\right)$, we obtain $O^{*}=(n v / b)\left(D^{*}+e\right)$. It follows from substituting this result back into the equation for $c E^{*}$ that $E^{*}=e n v / c$. To determine $D^{*}$, we take advantage of the fact that $R^{*}+O^{*}+E^{*}+A^{*}+D^{*}=1$. Thus,

$\left(\frac{n v}{a}+\frac{n v}{b}+\frac{n}{m}+1\right) D^{*}+e n v\left(\frac{1}{b}+\frac{1}{c}\right)=1$,

and upon rearrangement,

$D^{*}=\frac{\frac{1}{n v}-e\left(\frac{1}{b}+\frac{1}{c}\right)}{\left(\frac{1}{m}+\frac{1}{n}\right) \frac{1}{v}+\frac{1}{a}+\frac{1}{b}}$.

It is clear from the argument above that the equilibrium is feasible (i.e., nonnegative and sums to unity) and constitutes an IE (i.e., $A^{*}>0$ ) if and only if $D^{*}>0$, and by inspection we see that this latter inequality holds precisely when $\mathcal{R}_{0}>1$. Thus, an IE is feasible if and only if $\mathcal{R}_{0}>1$. Since $D^{*}$ must be of the form (A.7), and the other states are defined uniquely in terms of $D^{*}$, this argument also establishes that when the IE is feasible then it is unique.

For the remainder of this proof we assume that $\mathcal{R}_{0}>1$, so that the IE is feasible. Before we prove the stability of the IE, we first derive several useful identities and inequalities involving $D^{*}$. Again, we start with the relation $R^{*}+O^{*}+E^{*}+A^{*}+D^{*}=1$, but this time, we use the generic forms of $p\left(D^{*}\right)$ and $q\left(D^{*}\right)$ to obtain

$\left(\frac{n v}{a}+\frac{n}{m}+1\right) D^{*}+n v\left(\frac{1}{b}+\frac{q\left(D^{*}\right)}{c}\right) \frac{D^{*}}{p\left(D^{*}\right)}=1$.

We multiply both sides by $p\left(D^{*}\right) / n v$ and rearrange to get

$\left(\frac{1}{a}-\frac{1}{c}+\left(\frac{1}{m}+\frac{1}{n}\right) \frac{1}{v}\right) p\left(D^{*}\right) D^{*}+\left(\frac{1}{b}+\frac{1}{c}\right) D^{*}=\frac{p\left(D^{*}\right)}{n v}$.

In view of this equation, define

$\alpha=\frac{1}{b}+\frac{1}{c}, \quad \beta=\frac{1}{c}-\frac{1}{a}-\left(\frac{1}{m}+\frac{1}{n}\right) \frac{1}{v} \quad$ and $\quad \gamma=\frac{1}{n v}$.

Then $-\beta p\left(D^{*}\right) D^{*}+\alpha D^{*}=\gamma p\left(D^{*}\right)$ which implies that $p\left(D^{*}\right)=\alpha D^{*}$ । $\left(\beta D^{*}+\gamma\right)$. That is,

$p\left(D^{*}\right)=u\left(D^{*}\right)$,

where

$u(D) \stackrel{\text { def }}{=} \frac{\alpha D}{\beta D+\gamma}$.

Again, recall that $p(D)=D /(D+e)$. We observe that (i) $p(0)=u(0)=0$, (ii) $p^{\prime}(0)=1 / e>\alpha / \gamma=u^{\prime}(0)$ (because $\mathcal{R}_{0}>1$ ), (iii) $p(1)<1$, (iv) the equation $u(D)=1$ has a unique solution given by $D^{\prime}=\gamma /(\alpha-\beta)$, and (v) $D^{\circ} \in(0,1)$. Two cases arise. If $\beta \geq 0$, then $u(D)>0$ for $D>0$, which implies that $p(D)=u(D)$ for $0 \neq D \lesseqgtr D^{*}$. We conclude that $p^{\prime}\left(D^{*}\right)<u^{\prime}\left(D^{*}\right)$. Suppose now that $\beta<0$ and let $D^{+}=-\gamma / \beta \in\left(D^{\circ}, \infty\right)$. Then $u(D)>0$ for $D \in\left(0, D^{+}\right)$and $u(D)<0$ for $D>D^{+}$. In this case, $p(D) \gtreqless u(D)$ for $\left(0, D^{+}\right) \ni D \lesseqgtr D^{k}$. Again, we conclude that $p^{\prime}\left(D^{*}\right)<\mathfrak{u}^{\prime}\left(D^{*}\right)$. Thus, in either situation we have

$p^{\prime}\left(D^{*}\right)<u^{\prime}\left(D^{*}\right)$.

Finally, it is clear from the form of $p(D)=D /(D+e)$ that

$p^{\prime}\left(D^{*}\right)<\frac{p\left(D^{*}\right)}{D^{*}}$
This inequality, (A.6), and the condition $\mathcal{R}_{0}>1$ together imply that

$p^{\prime}\left(D^{*}\right) b O^{*}<n v<p^{\prime}(0) b \bar{O}$.

Suppose now that $a \geq c$ or $b+c \geq \max \{m, n\}$. To examine the stability of the IE, we linearize system (2.1) around the IE to produce the Jacobian matrix

$J^{*}=\left(\begin{array}{ccccc}-a & 0 & 0 & 0 & v n \\ a & -b & c & 0 & 0 \\ 0 & 0 & -c & 0 & 0 \\ 0 & 0 & 0 & -m & w n \\ 0 & 0 & 0 & m & -n\end{array}\right)+\left(\begin{array}{ccccc}0 & 0 & 0 & 0 & 0 \\ 0 & 0 & 0 & 0 & 0 \\ 0 & q\left(D^{*}\right) b & 0 & 0 & q^{\prime}\left(D^{*}\right) b O^{*} \\ 0 & p\left(D^{*}\right) b & 0 & 0 & p^{\prime}\left(D^{*}\right) b O^{*} \\ 0 & 0 & 0 & 0 & 0\end{array}\right)$.

This time, it is not helpful to express the complicated eigenvalues of the matrix $J^{*}$ explicitly. However, according to the Gershgorin circle theorem (Varga, 2004), the eigenvalues of $J^{*}$ lie within the region of the complex plane defined by the union of the closed disks $D(-a, a)$ $D(-b, b), D(-c, c), D(-m, m)$, and $D\left(-n, n+2 p^{\prime}\left(D^{*}\right) b O^{*}\right)$. Unfortunately, the final disk intrudes somewhat upon the right-half plane and so we cannot rule out the possibility that some eigenvalues of $J^{*}$ may reside there. (In fact, $J^{*}$ has a simple zero eigenvalue, but this is to be expected because we are only interested in perturbations satisfying $R+O+E+A+D=1$.

Although this particular approach has provided some insight into the possible location of eigenvalues, let us abandon it and instead write system (2.1) as the reduced system (A.1) in the proof of Theorem 1. Linearizing around the IE results in the Jacobian matrix

$$
\begin{aligned}
K^{*} & =\left(\begin{array}{cc|cc}
-a & 0 & 0 & n v \\
a-c & -b-c & -c & -c \\
\hline & -m & n w \\
m & -n
\end{array}\right) \\
& +\left(\begin{array}{cc|cc} 
& \\
\hline 0 & p\left(\mathrm{D}^{*}\right) b & 0 & p^{\prime}\left(\mathrm{D}^{*}\right) b 0^{*} \\
0 & 0 & 0 & 0
\end{array}\right) .
\end{aligned}
$$

The eigenvalues of $K^{*}$ are still complicated, and now the Gershgorin circle theorem provides less information about their locations in the complex plane. But, it can be noted that the characteristic polynomial $s(\hat{\lambda})$ of $K^{*}$ divides the characteristic polynomial of $J^{*}$ with quotient $-\lambda$ (two different computer algebra packages confirm this result). Thus, the nonzero eigenvalues of $J^{*}$ are exactly the eigenvalues of $K^{*}$.

We now show that the IE is locally asymptotically stable by establishing that the characteristic polynomial of $K^{*}$ is stable (i.e., its zeros have negative real parts). The polynomial $s(\lambda)=\lambda^{4}$ $+\omega_{1} \lambda^{3}+\omega_{2} \lambda^{2}+\omega_{3} \lambda+\omega_{4}$ is given by

$s(\lambda)=\operatorname{det}\left(\lambda I-K^{*}\right)=\left|\begin{array}{cccc}\lambda+a & 0 & 0 & -n v \\ c-a & \lambda+b+c & c & c \\ 0 & -p\left(D^{*}\right) b & \lambda+m & -n w-p^{\prime}\left(D^{*}\right) b O^{*} \\ 0 & 0 & -m & \lambda+n\end{array}\right|$

Expanding the determinant along the first row, we obtain

$s(\lambda)=(\lambda+a)(\lambda+b+c)\left[(\lambda+m)(\lambda+n)-m\left(n w+p^{\prime}\left(D^{*}\right) b O^{*}\right)\right]$

$+(\lambda+a)(\lambda+m+n) p\left(D^{*}\right) b c+(c-a) m n v p\left(D^{*}\right) b$.

Consequently, the coefficients of $s(\lambda)$ are

$\omega_{1}=a+b+c+m+n$,

$\omega_{2}=a(b+c+m+n)+(b+c)(m+n)+b c p\left(D^{*}\right)+m\left(n v-p^{\prime}\left(D^{*}\right) b O^{*}\right)$,

$\omega_{3}=a(b+c)(m+n)+(a+m+n) b c p\left(D^{*}\right)+(a+b+c) m\left(n v-p^{\prime}\left(D^{*}\right) b O^{*}\right)$.

$\omega_{4}=(a c(m+n)+(c-a) m n v) b p\left(D^{*}\right)+a(b+c) m\left(n v-p^{\prime}\left(D^{*}\right) b O^{*}\right)$. 
Observe from the positivity of $p\left(D^{*}\right)$ and (A.13) that $\omega_{1}, \omega_{2}$, and $\omega_{3}$ are all positive. As for $\omega_{4}$, we in turn employ (A.8), (A.6), (A.9), (A.11) and (A.10) to compute

$$
\begin{aligned}
\omega_{4}= & a b c m n v\left[-\beta p\left(D^{*}\right)+\alpha\left(1-p^{\prime}\left(D^{*}\right) \frac{D^{*}}{p\left(D^{*}\right)}\right)\right] \\
& >a b c m n v\left[-\beta u\left(D^{*}\right)+\alpha\left(1-u^{\prime}\left(D^{*}\right) \frac{D^{*}}{u\left(D^{*}\right)}\right)\right] \\
= & a b c m n v\left[-\frac{\beta \alpha D^{*}}{\beta D^{*}+\gamma}+\alpha\left(1-\frac{\gamma}{\beta D^{*}+\gamma}\right)\right] \\
= & 0 .
\end{aligned}
$$

Thus, $\omega_{4}>0$. Since $s(\lambda)$ has positive coefficients, it has no nonnegative real zeros. We now show that no zero of $s(\lambda)$ (either real or complex) has nonnegative real part. We use the fact that the polynomial $s(\lambda)$ is stable if and only if the Routh-Hurwitz determinants

$$
\begin{aligned}
\Delta_{1} & =\left|\omega_{1}\right|, \quad \Delta_{2}=\left|\begin{array}{cc}
\omega_{1} & \omega_{3} \\
1 & \omega_{2}
\end{array}\right|, \quad \Delta_{3}=\left|\begin{array}{ccc}
\omega_{1} & \omega_{3} & 0 \\
1 & \omega_{2} & \omega_{4} \\
0 & \omega_{1} & \omega_{3}
\end{array}\right|, \text { and } \\
\Delta_{4} & =\left|\begin{array}{cccc}
\omega_{1} & \omega_{3} & 0 & 0 \\
1 & \omega_{2} & \omega_{4} & 0 \\
0 & \omega_{1} & \omega_{3} & 0 \\
0 & 1 & \omega_{2} & \omega_{4}
\end{array}\right|
\end{aligned}
$$

are all positive (Gantmacher, 1960). Clearly, $\Delta_{1}=a+b+c+m+n$ is positive and by (A.13),

$$
\begin{aligned}
\Delta_{2}= & (a+b+c)(a+m+n)(b+c+m+n)+(b+c) b c p\left(D^{*}\right) \\
& +m(m+n)\left(n v-p^{\prime}\left(D^{*}\right) b O^{*}\right)
\end{aligned}
$$

is also positive. Since $\Delta_{4}=\omega_{4} \Delta_{3}$, it remains only to show that $\Delta_{3}=\omega_{3} \Delta_{2}-\omega_{4} \omega_{1}^{2}$ is positive. In view of (A.14) and the form of $\Delta_{2}$ above, it is helpful to write

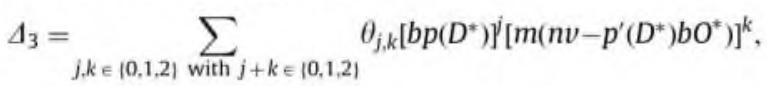

where the bracketed quantities are both positive and

$$
\begin{aligned}
\theta_{0,0}= & a(a+b+c)(a+m+n)(b+c)(b+c+m+n)(m+n), \\
\theta_{1,0}= & c(a+b+c)(a+m+n)^{2}(b+c+m+n)+a c(b+c)^{2}(m+n) \\
& -a c(a+b+c+m+n)^{2}(m+n)+m n v(a+b+c+m+n)^{2}(a-c), \\
\theta_{0,1}= & (a+b+c)^{2}(a+m+n)(b+c+m+n)+a(b+c)(m+n)^{2} \\
& -a(a+b+c+m+n)^{2}(b+c), \\
\theta_{2,0}= & c^{2}(a+m+n)(b+c), \\
\theta_{1,1}= & c(a+b+c)(b+c)+c(a+m+n)(m+n), \\
\theta_{0,2}= & (a+b+c)(m+n) .
\end{aligned}
$$

We remark that $\theta_{0,0}, \theta_{2,0}, \theta_{1,1}$, and $\theta_{0,2}$ are clearly positive. Furthermore, since we can write

$\theta_{0.1}=\left[a^{2}+a(m+n)+(b+c)(b+c+m+n)\right](a+b+c)(m+n)$

we also find that $\theta_{0,1}$ is positive. Lastly, we decompose $\theta_{1,0}=\theta_{1,0}^{+}+\theta_{1,0}^{*}$, where

$\theta_{1,0}^{+}=\left[a^{2}+a(b+c)+(b+c+m+n)(m+n)\right] c(a+m+n)(b+c)$,

$\theta_{1,0}^{*}=m n v(a+b+c+m+n)^{2}(a-c)$.

It is clear that $\theta_{1,0}^{+}>0$. If $a \geq c$, then we also obtain $\theta_{1,0}^{*} \geq 0$, and therefore that $\theta_{1.0}>0$. It now follows by inspection that $\Delta_{3}$ is positive, as desired. Suppose however that $a<c$. Then by assumption $b+c \geq \max \{m, n\}$. In this case,

$$
\begin{aligned}
\Delta_{3} & >\theta_{0,0}+\theta_{1,0} b p\left(D^{*}\right)=\theta_{0,0}+\left(\theta_{1,0}^{+}+\theta_{1,0}^{*}\right) b p\left(D^{*}\right) \\
& \geq\left(\theta_{0,0} v+\theta_{1,0}^{+} v b+\theta_{1,0}^{*} b\right) p\left(D^{*}\right) .
\end{aligned}
$$

It suffices to show that $\theta_{0.0} v+\theta_{1.0}^{+} v b+\theta_{1,0}^{*} b \geq 0$. According to Mathematica 6.0 , this quantity can be expressed as a cubic polynomial $\psi(a)=\xi_{0}+\xi_{1} a+\xi_{2} a^{2}+\xi_{3} a^{3}$ with

$\xi_{0}=b c v(b+c+m+n)\left((b+c)\left(m^{2}+m n+n^{2}\right)-(m+n) m n\right)$

and $\xi_{1}, \xi_{2}$, and $\xi_{3}$ being positive by inspection. Since $b+c \geq \max \{m, n\}$, we conclude that $\psi(a) \geq 0$ and hence that $\Delta_{3}$ is positive. This completes the proof.

\section{References}

Beekman, M., Fathke, R.L., Seeley, T.D., 2006. How does an informed minority of scouts guide a honey bee swarm as it flies to its new home. Anim. Behav. 71, 161-171.

Britton, N.F., Franks, N.R., Pratt, S.C., Seeley, T.D., 2002. Deciding on a new home: how do honey bees agree? Proc. R. Soc. Lond. B 269, 1383-1388.

Camazine, S., Visscher, P.K., Finley, J., Vetter, R.S., 1999. House-hunting by honey bee swarms: collective decisions and individual behaviors. Insectes. Soc. 46, 348-360. Franks, N.R., Dornhaus, A., Fitzsimmons, J.P., Stevens, M., 2003. Speed versus accuracy in collective decision making. Proc. R. Soc. Lond. B 270, 2457-2463.

Gantmacher, F.R., 1960. The Theory of Matrices, vol II, Chelsea Pub. Co., New York, New York

Janson, S., Middendorf, M., Beekman, M., 2007. Searching for a new home-scouting behavior of honeybee swarms. Behav. Ecol. 18, 384-392.

Mallon, E.B., Pratt, S.C., Franks, N.R., 2001. Individual and collective decisionmaking during nest site selection by the ant Leptothorax albipennis. Behav. Ecol. Sociobiol. 50, 352-359.

Marshall, J.A.R., Bogacz, R., Dornhaus, A., Planqué, R., Kovacs, T., Franks, N.R., 2009. on optimal decision making in brains and social insect colonies. J. R. Soc. Interface 6, 959-1102.

Myerscough, M.R., 2003. Dancing for a decision: a matrix model for nest-site choice by honey bees. Proc. R. Soc. Lond. B 270, 577-582.

Passino, K.M., Seeley, T.D., 2006. Modeling and analysis of nest-site selection by honey bee swarms: the speed and accuracy trade-off. Behav. Ecol. Sociobiol. 59 (3), 427-442.

Passino, K.M., Seeley, T.D., Visscher, P.K., 2008. Swarm cognition in honey bees. Behav. Ecol. Sociobiol. 62 (3), 401-414.

Perdriau, B.S., Myerscough, M.R., 2007. Making good choices with variable information: a stochastic model for nest-site selection by honeybees. Biol. Lett. 3, 140-143.

Pratt, S.C., 2005. Quorum sensing by encounter rates in the ant Temnothorax albipennis. Behav. Ecol. 16, 488-496.

Pratt, S.C., Mallon, E.B., Sumpter, D.J.T., Franks, N.R., 2002. Quorum sensing, recruitment, and collective decision-making during colony emigration by the ant Leptothorax albipennis. Behav. Ecol. Sociobiol. 52, 117-127.

Pratt, S.C., Sumpter, D.J.T., Mallon, E.B., Franks, N.R., 2005. An agent-based model of collective nest choice by the ant Temnothorax albipennis. Anim. Behav. 70, 1023-1036.

Schultz, K.M., Passino, K.M., Seeley, T.D., 2008. The mechanism of flight guidance in honeybee swarms: subtle guides or streaker bees? J. Exp. Biol. 211, 3287-3295.

Seeley, T.D., 2003. Consensus building during nest-site selection in honey bee swarms: the expiration of dissent. Behav. Ecol. Sociobiol. 53, 417-424.

Seeley, T.D., Buhrman, S.C., 1999. Group decision making in swarms of honey bees. Behav. Ecol. Sociobiol. 45, 19-31.

Seeley, T.D., Buhrman, S.C., 2001. Nest-site selection in honey bees: how well do swarms implement the "best-of-n" decision rule? Behav. Ecol. Sociobiol. 49, 416-427.

Seeley, T.D., Visscher, P.K., 2003. Choosing a home: how the scouts in a honey bee swarm perceive the completion of their group decision making. Behav. Ecol. Sociobiol. 54, 511-520.

Seeley, T.D., Visscher, P.K., 2004. Group decision making in nest-site selection by honey bees. Apidologie 35, 1-16.

Seeley, T.D., Visscher, P.K., 2004. Quorum sensing during nest-site selection by honey bee swarms. Behav. Ecol. Sociobiol. 56, 594-601

Seeley, T.D., Visscher, P.K., Passino, K.M., 2006. Group decision making in honey bee swarms. Am. Sci. 94 (3), 220-229.

Smith, H.L., Waltman, P., 1995. The Theory of the Chemostat: Dynamics of Microbial Competition. Cambridge University Press, Cambridge.

van den Driessche, P., Watmough, I., 2002. Reproduction numbers and subthreshold endemic equilibria for compartmental models of disease transmission. Math. Biosci. 180, $29-48$.

Varga, R.S., 2004. Gersgorin and His Circles. Springer, Berlin. 